\title{
ENFERMEDAD ARTICULAR DEGENERATIVA Y CAMBIOS ENTESIALES EN SEIS COLECCIONES ÓSEAS PREHISPÁNICAS DEL NOROCCIDENTE DE AMÉRICA DEL SUR
}

\author{
DEGENERATIVE JOINT DISEASE AND ENTHESEAL CHANGES \\ IN SIX PRE-COLUMBIAN SKELETAL COLLECTIONS FROM \\ THE NORTHWEST OF SOUTH AMERICA
}

\author{
Claudia Mercedes Rojas-Sepúlveda ${ }^{1,2}$ y Olivier Dutour ${ }^{3,4}$
}

\begin{abstract}
La enfermedad articular degenerativa (EAD) y los cambios entesiales (CE) fueron estudiados en seis colecciones óseas prehispánicas del noroeste de América del Sur con el objetivo de aportar al debate sobre las relaciones entre estos indicadores y la actividad física, así como al conocimiento sobre las condiciones de vida de los pueblos prehispánicos de la región. Se estudiaron tres series del altiplano central de Colombia (Soacha, Marín y Tunja-UPTC), dos series de Panamá (Panamá Viejo y Sitio Sierra) y una colección de la costa central peruana (Ancón 1). El uso de una metodología estandarizada permitió la comparación directa de frecuencias y perfiles de EAD y de CE. Se observó una importante variedad de perfiles y de frecuencias en las diferentes colecciones óseas, pero también algunas tendencias similares en colecciones de ambientes semejantes. Se observó una falta de correspondencia entre los resultados de la EAD y de los CE que podría indicar que, a pesar de que tienen una etiología multifactorial, podrían ser respuestas a diferentes tipos de actividad: una actividad repetitiva podría relacionarse más con la EAD, mientras que una actividad que incluya cargas pesadas podría relacionarse más con los CE.
\end{abstract}

Palabras claves: actividad física, paleoepidemiología, osteoartritis, entesopatías, marcadores óseos de actividad, MSM, bioarqueología.

Degenerative joint disease (DJD) and entheseal changes (EC) were studied in six Pre-Hispanic skeletal collections from the northwest of South America, in order to give insight both into their relationship with physical activity and into life conditions in this region during Pre-Columbian times. Three series from the highlands of Colombia (Soacha, Marín and Tunja-UPTC), two series from Panama (Panamá Viejo and Sitio Sierra) and one from the Central Coast of Peru (Ancón 1) were analyzed for this study. The use of a standardized methodology allowed direct comparisons between the frequencies and the profiles of DJD and EC. An important variability in profiles and frequencies was observed between series. However, it was also possible to observe several similar trends in series from analogous environments. There is a mismatch between results from DJD and EC which may indicate that, despite having a multi-factorial aetiology, they may respond to different types of physical activity: repetitive activity would be more related to DJD, while activities involving heavy loads would be more connected with EC.

Key words: Physical activity, paleoepidemiology, osteoarthritis, enthesopathies, musculoskeletal stress markers, MSM, bioarchaeology.

La relación entre la enfermedad articular degenerativa-EAD, también llamada osteoartrosis, y la actividad física ha sido discutida en antropología por más de treinta años. Algunos investigadores han mostrado una conexión entre la EAD y un estilo de vida exigente desde el punto de vista biomecánico (Bridges 1991, 1992; Cope et al. 2005; Crubézy et al.
2002; Jurmain 1977; 1980; 1990; Jurmain y Kilgore 1995; Klaus et al. 2009; Larsen 1987; Lieverse et al. 2007; Lovell y Dublenko 1999; Molnar et al. 2011; Ortner 2003; Peyron 1986; Resnick 2002; Rogers y Waldron 1995; Rogers et al. 1987; Ubelaker 1989; 1989; Waldron 1997; Walker y Hollimon 1989; Watkins 2012). Sin embargo, algunos otros han

1 Unité d'Anthropologie Bioculturelle-UMR 7268, Université de la Méditerranée/CNRS/EFS, Marseille, Francia, 13344. clarosepul@gmail.com

2 Programa de Antropología, Facultad de Humanidades, Universidad del Magdalena, Santa Marta, Magdalena, Colombia, 47004. Dirección postal: Calle 24A 75-10. Modelia, Bogotá, Colombia.

3 École Pratique des Hautes Études-Laboratoire de Paléoanthropologie-UMR 5199/PACEA/CNRS/Université de Bordeaux 1 Talence, Francia, 33405. dutour27@gmail.com

4 Department of Anthropology, University of Toronto, Toronto, ON, Canada, M5S 2S2. 
encontrado una fuerte correlación entre la EAD y la edad (Bourke 1967; Jurmain y Kilgore 1995; Molnar et al. 2011); considerándola como simple consecuencia del proceso de envejecimiento y de la bipedación característica del ser humano, en particular cuando se observa sobre la columna vertebral (Knüsel et al. 1997).

La EAD es la degeneración del cartílago y del hueso articular, es asimétrica y pluriarticular, no inflamatoria, crónica y causa la deformación de la articulación asociando pérdida y formación ósea (Bourke 1967; Dutour y Ardagna 2005; Resnick 2002; Rothschild 1997). La EAD es multifactorial, se relaciona con la edad, el sexo, el trauma, la genética, el bipedalismo, la obesidad, la nutrición, el estrés biomecánico y la actividad física (Bridges 1992; Campillo 2001; Cunha 2003; Goodman y Martin 2002; Goodman et al. 1984; Jurmain 1977, 1980; Jurmain y Kilgore 1995; Larsen 1987; Malgosa 2003; Ortner 2003; Peyron 1986; Resnick 2002; Robledo et al. 1993; Rogers y Dieppe 1994; Rogers y Waldron 1995; Rogers et al. 1987; Ubelaker 1989; Weiss y Jurmain 2007).

La EAD es frecuentemente observada en restos óseos de sitios arqueológicos de gran antigüedad, pero produce consecuencias socioeconómicas aún en la actualidad, como incapacidad para laborar, hospitalización, invalidez de corta y larga duración, cirugías, entre otras (Bridges 1992; Cohen y Armelagos 1984; Goodman y Martin 2002; Jurmain y Kilgore 1995; Kjeken et al. 2004; Ortner 2003; Peyron 1986; Rogers y Dieppe 1990; Thillaud 1992; Waldron 1997; Weiss y Jurmain 2007). La paleoepidemiología de la EAD ha sido estudiada en varias colecciones óseas de Norteamérica (Bourke 1967; Bridges 1991, 1992; Dabbs 2011; Jurmain 1977, 1980, 1990, Jurmain y Kilgore 1995; Ortner 1968; Walker y Hollimon 1989; Watkins 2012; Woo y Sciulli 2011), Europa (Berato et al. 1990; Crubézy et al. 2002; Molnar et al. 2011; Rogers y Dieppe 1994; Rogers et al. 1997; H. Waldron 1991, 1997; T. Waldron 1992, 1997) y Asia (Baker et al. 2012; Cope et al. 2005; Eshed et al. 2010; Inoue et al. 2001; Lieverse et al. 2007). Sin embargo, las diferencias en las metodologías utilizadas dificultan la comparación de los resultados. El estudio de la paleoepidemiología de la EAD es muy reciente en colecciones óseas de América del Sur (Arrieta y Mendonça 2011; Klaus et al. 2009; Pechenkina y Delgado 2006; Ponce 2010; Rojas-Sepúlveda et al. 2008).
Los cambios entesiales (CE) (Henderson y AlvesCardoso 2013; Jurmain y Villotte 2010; Villotte y Knüsel 2013), llamados tradicionalmente marcadores óseos de actividad (MOA) y musculoskeletal stress markers (MSM), son modificaciones producidas en los sitios de inserción y origen de los músculos y los tendones. En antropología se han estudiado los marcadores de estrés ocupacional, los cuales incluyen los marcadores de estrés musculoesquelético y los marcadores de robustez (Jurmain y Villotte 2010; Kennedy 1998; Santos et al. 2010). En torno a los $\mathrm{CE}$, se ha dado una activa discusión entre investigadores en los últimos años, en particular en lo que respecta a la terminología y las metodologías de estudio (Henderson y Alves-Cardoso 2013; Jurmain y Villotte 2010; Villotte y Knüsel 2013). Los marcadores óseos de actividad se han definido como lesiones óseas localizadas en los sitios de inserción de los músculos y los tendones, causados por su prolongada hiperactividad (Dutour 1986); o como las marcas producidas por los músculos, los ligamentos o los tendones cuando se insertan en el hueso (Hawkey y Merbs 1995). Sin embargo, el término "cambio entesial" (entheseal change) ha sido propuesto recientemente por ser más genérico y más neutral para designar a las alteraciones de las entesis observadas en material óseo (Jurmain y Villotte 2010), teniendo en cuenta que dichos cambios pueden ser originados por varias causas y no solamente por la actividad física. Si bien la actividad es solo uno de esos factores, el estudio de estos marcadores ha sido interesante porque ofrece la oportunidad de acercase al uso de un músculo, ya que la plasticidad del hueso responde al estrés muscular por medio de un cambio morfológico (Hawkey 1998; Hawkey y Merbs 1995; Jurmain y Villotte 2010; Kennedy 1998; Mariotti et al. 2004; 2007; Molnar 2006; 2008; Munson Chapman 1997; Oumaoui et al. 2004; Peterson 1998; Robb 1998; Santos et al. 2010; Steen y Lane 1998; Stirland 1998; Villotte 2008; Weiss 2003a; 2004; Wilczak 1998). Si un sitio de inserción está bajo estrés con frecuencia, el número de capilares se aumenta en el periostio, lo cual estimula la remodelación de osteones, produciendo una hipertrofia ósea y una inserción muscular rugosa (Hawkey y Merbs 1995; Munson Chapman 1997). El aumento de los capilares resulta del engrosamiento del diámetro de las fibras musculares por la producción de miofibrillas, mitocondrias y retículo sarcoplásmico (Steen y Lane 1998). Los CE han sido estudiados principalmente para reconstruir 
los estilos de vida en el pasado en Europa (Crubézy et al. 2002; Havelková et al. 2011; Molnar 2006, 2008; Molnar et al. 2011; Niinimäki 2011; Palfi y Dutour 1996; Robb 1994, 1998; Stefanović y Porčić 2011; Stirland 1998; Villotte 2006, 2008; Villotte et al. 2010), Norteamérica (Lovell y Dublenko 1999; Munson Chapman 1997; Steen y Lane 1998; Weiss 2003b, 2004, 2007; Weiss et al. 2012), Asia (Baker et al. 2012; Eshed et al. 2004; Peterson 1998), África (Churchill y Morris 1998; Dutour 1986) y recientemente, en América del Sur (Acosta 2012; Ponce 2010). Diversos aspectos metodológicos con respecto al registro de la información y al tratamiento estadístico, así como a los factores que influyen en la presencia de los CE han sido objeto de discusión durante las últimas décadas (Cardoso y Henderson 2010; Churchill y Morris 1998; Hawkey y Merbs 1995; Henderson y Alves-Cardoso 2013; Jurmain y Villotte 2010; Mariotti et al. 2004, 2007; Molnar et al. 2011; Niinimäki 2011; Robb 1998; Santos et al. 2010; Stefanović y Porčić 2011; Stirland 1998; Villotte 2006, 2008; Villotte et al. 2010; Villotte y Knüsel 2013; Weiss 2003b, 2004, 2007; Weiss et al. 2012; Wilczak 1998).
Este estudio busca contribuir a la interpretación de la información sobre poblaciones del pasado con respecto a la enfermedad articular degenerativa y los cambios entesiales. De esta manera, busca aportar al debate sobre la relación entre actividad física, EAD y CE.

\section{Materiales y Métodos}

La EAD y los CE fueron estudiados en seis colecciones óseas prehispánicas: Soacha (en algunos casos referida como Soacha Portoalegre, Partalegre o Portobelo), Marín y Tunja-UPTC (también conocida como Cercado grande de los Santuarios) en Colombia; Panamá Viejo y Sitio Sierra en Panamá; y Ancón 1 en Perú (Figura 1, Tablas 1 y 2). Aquellos individuos de las colecciones que tuvieran un estado de conservación aceptable y fueran mayores de 15 años fueron incluidos en el estudio.

Un observador (Rojas-Sepúlveda) realizó el registro completo de la información con el fin de evitar errores interobservador. El sexo y la edad de los individuos fueron estimados siguiendo métodos antropológicos convencionales (Brothwell 1965;

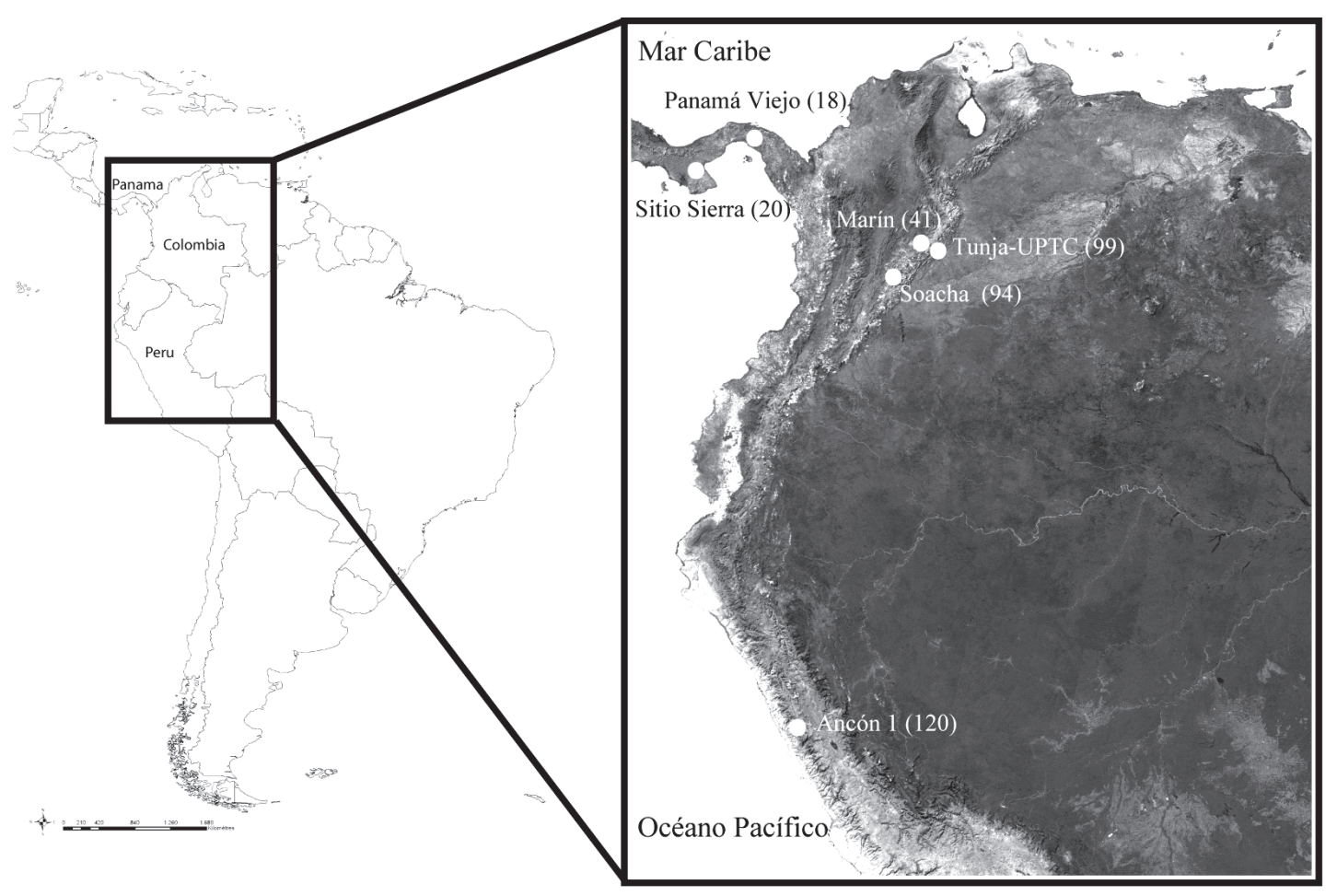

Figura 1. Colecciones óseas estudiadas.

Skeletal series studied. 
Tabla 1. Información sobre las colecciones estudiadas en Colombia. Information about the series studied in Colombia.

\begin{tabular}{|c|c|c|c|}
\hline & Soacha $^{1}$ & Marín & Tunja-UPTC $^{2}$ \\
\hline Ambiente & $\begin{array}{l}\text { Altitud: } 2565 \mathrm{msm} . \\
\text { Temperatura: } 13^{\circ} \mathrm{C} \text {. } \\
\text { Rico en recursos }\end{array}$ & $\begin{array}{c}\text { Altitud: } 2600 \mathrm{msm} \text {. } \\
\text { Temperatura: } 12-14^{\circ} \mathrm{C} \text {. } \\
\text { Lacustre }\end{array}$ & $\begin{array}{l}\text { Altitud: } 2800 \mathrm{msm} . \\
\text { Temperatura: } 13^{\circ} \mathrm{C} \text {. } \\
\text { Rico en recursos }\end{array}$ \\
\hline Cultura & Muisca de Bogotá (Sur) & Muisca de Boyacá (Norte) & Muisca de Boyacá (Norte) \\
\hline Cronología del sitio & 700-1600 d.C. & 700-1600 d.C. & I-XVIII d.C. \\
\hline Cronología colección & XI-XIII d.C. & XIII-XIV d.C. & VIII-XVIII d.C. \\
\hline Número de tumbas & 133 & 43 & 285 \\
\hline $\begin{array}{l}\text { Actividades de } \\
\text { subsistencia }\end{array}$ & $\begin{array}{l}\text { Agricultura, caza, pesca, } \\
\text { manufactura, recolección, } \\
\text { minería, comercio }\end{array}$ & $\begin{array}{l}\text { Agricultura, caza, pesca, } \\
\text { recolección, manufactura }\end{array}$ & $\begin{array}{l}\text { Manufactura, caza, pesca, } \\
\text { comercio, agricultura }\end{array}$ \\
\hline Sistema político & $\begin{array}{l}\text { Confederación de cacicazgos, } \\
\text { poder religioso y político }\end{array}$ & $\begin{array}{c}\text { Confederación de cacicazgos, } \\
\text { poder religioso y político }\end{array}$ & $\begin{array}{c}\text { Confederación de cacicazgos, } \\
\text { poder religioso y político. }\end{array}$ \\
\hline Sistema social & $\begin{array}{l}\text { Estratificación social. Colección } \\
\text { integrada por gente del común y } \\
\text { guerreros }\end{array}$ & $\begin{array}{c}\text { Estratificación. Contextos } \\
\text { funerarios no típicos de la zona } \\
\text { muisca }\end{array}$ & Probable estratificación \\
\hline $\begin{array}{c}\text { Estudios } \\
\text { bioantropológicos }\end{array}$ & $\begin{array}{l}\text { (Martínez 2005; Rodríguez } \\
\text { 1994, 1999; Rojas-Sepúlveda } \\
\text { 2005; Rojas-Sepúlveda et al. } \\
\text { 2008) }\end{array}$ & $\begin{array}{c}\text { Gonzalo Correal y Hernando } \\
\text { José Umaña (Boada 1987, } \\
\text { 1988; Martínez 2005) }\end{array}$ & $\begin{array}{c}\text { (Álvarez y Rodríguez 2001; } \\
\text { Rodríguez 2006). }\end{array}$ \\
\hline $\begin{array}{l}\text { Estado de conservación } \\
\text { Efectivo estudiado }\end{array}$ & $\begin{array}{l}\text { Buen estado general } \\
91 \text { individuos }\end{array}$ & $\begin{array}{l}\text { Buen estado general } \\
41 \text { individuos }\end{array}$ & $\begin{array}{l}\text { Estado aceptable } \\
99 \text { individuos }\end{array}$ \\
\hline
\end{tabular}

${ }^{1}$ En algunos casos referida como Soacha Portoalegre, Portalegre o Portobelo; ${ }^{2}$ También conocido como Cercado grande de los Santuarios.

Tabla 2. Información sobre las colecciones estudiadas en Panamá y en Perú. Information about the series studied in Panamá and Peru.

\begin{tabular}{|c|c|c|c|}
\hline & \multicolumn{2}{|c|}{ Panamá } & \multirow{2}{*}{$\begin{array}{c}\text { Perú } \\
\text { Ancón } 1\end{array}$} \\
\hline & Panamá Viejo & Sitio Sierra & \\
\hline Ambiente & $\begin{array}{l}\text { Altitud: } 0 \text { msm. Bosque tropical. } \\
\text { Recursos Marinos. Inundaciones } \\
\text { por el fenómeno de El Niño }\end{array}$ & $\begin{array}{c}\text { Altitud: } 300 \mathrm{msm} \text {. Bosque } \\
\text { tropical. Recursos Marinos. } \\
\text { Variaciones por el fenómeno de } \\
\text { El Niño }\end{array}$ & $\begin{array}{c}\text { Altitud: } 0 \text { msm. Recursos } \\
\text { Marinos. Suelo árido. } \\
\text { Inundaciones por el fenómeno } \\
\text { de El Niño }\end{array}$ \\
\hline Cultura & Gran Darién (Cuevas) & Gran Coclé & Huaura y Chancay \\
\hline Cronología del sitio & $\begin{array}{l}\text { 1420-490 a.p. } 530-1460 \text { d.C. } \\
\text { IX-XV d.C. }\end{array}$ & 400 a.C. -600 d.C. & 2500 a.C. -1534 d.C. \\
\hline Cronología colección & $\begin{array}{l}\text { 1420-490 a.p. 530-1460 d.C. } \\
\text { IX-XV d.C. }\end{array}$ & 40 d.C-350 d.C. I-IV d.C. & $\begin{array}{l}\text { Horizonte Medio. 600-1000 d.C. } \\
\text { VII-XI d.C. }\end{array}$ \\
\hline Número de tumbas & 65 & $24 ?$ & Misión 1950-1953: 875 tumbas \\
\hline $\begin{array}{l}\text { Actividades de } \\
\text { subsistencia }\end{array}$ & $\begin{array}{l}\text { Agricultura, pesca, recolección } \\
\text { de moluscos, manufactura }\end{array}$ & $\begin{array}{l}\text { Pesca, caza, agricultura, } \\
\text { comercio, manufactura }\end{array}$ & $\begin{array}{c}\text { Comercio, pesca, recolección de } \\
\text { moluscos }\end{array}$ \\
\hline Sistema político & Cacicazgos & Cacicazgos & Actividad militar \\
\hline Sistema social & Probable estratificación & $\begin{array}{c}\text { Probable estratificación, la } \\
\text { colección es de gente del común }\end{array}$ & $\begin{array}{l}\text { Estratificación, la colección es } \\
\text { de gente del común }\end{array}$ \\
\hline $\begin{array}{l}\text { Estudios } \\
\text { bioantropológicos }\end{array}$ & (Martín et al. 2009) & (Norr 1991; Nowàcka 1989) & $\begin{array}{l}\text { (Rhode y Benfer 2006; Rojas- } \\
\text { Sepúlveda y Dutour 2009; } \\
\text { Slovak 2007). }\end{array}$ \\
\hline Estado de conservación & Mala conservación & Buena conservación & Buena conservación \\
\hline Efectivo estudiado & 18 individuos & 19 individuos & 116 individuos \\
\hline
\end{tabular}


Buikstra y Ubelaker 1994; Isçan et al. 1984; Isçan et al. 1985; Suchey y Brooks 1988; White y Folkens 2000). Las anomalías óseas fueron observadas (Larsen 1987; 1999; Ortner 2003; Thillaud 1992, 1994) con el fin de descartar aquellos individuos que presentaran signos de DISH (Diffuse idiopathic skeletal hiperostosis), espondilitis anquilosante o trauma de gran proporción (Benjamin et al. 2006; Dutour 1992; Hawkey y Merbs 1995).

Se registró el estado de conservación de cada una de las articulaciones (completa: más del $75 \%$ de la articulación observable; suficientemente completa: 50-75\%; incompleta: 25-50\%; muy incompleta: menos del 25\%; y no observable). Se observaron de manera macroscópica las diferentes manifestaciones de la EAD: osteofitos (Nathan 1962; Rogers y Waldron 1995), labiación (Rogers et al. 1987; Rojas-Sepúlveda et al. 2008), eburnación (Bridges 1992; Ortner 2003; Rogers y Waldron 1995; Rogers et al. 1987; Rothschild 1997) y porosidad (Rothschild 1997). Dichas manifestaciones se registraron por medio de códigos relacionados con la expresión de la manifestación (Jurmain 1990; Nathan 1962; Rojas-Sepúlveda et al. 2008): de 0 (ausente) a 3 (extrema) (Rojas-Sepúlveda et al. 2008).

Los CE fueron observados en los sitios de inserción u origen de músculos o ligamentos descritos en la Tabla 3. Se utilizó la metodología propuesta por Hawkey y Merbs (1995), en la cual se definen tres categorías: el marcador de robustez (rugosidad), la lesión causada por estrés (porosidad hasta una laguna) y la exostosis osificante. Cada una de ellas se codificó de 0 (expresión ausente) a 3 (expresión fuerte). El código no observable se utilizó cuando el sitio de inserción no era visible. Los marcadores de robusticidad y las lesiones por estrés son un continuum (Hawkey y Merbs 1995), los códigos variaron entonces de 0 a 6 . Ya que las exostosis osificantes se relacionan con trauma, no fueron consideradas. Este método es simple, ha sido utilizado en muchos estudios (Eshed et al. 2004; Lovell y Dublenko 1999; Molnar 2006, 2008; Molnar et al. 2011; Munson Chapman 1997; Niinimäki 2011; Peterson 1998; Steen y Lane 1998; Weiss 2003b, 2004) y produce niveles aceptables de error de observación (Hawkey y Merbs 1995). Si bien otras propuestas han visto la luz (Henderson y Alves-Cardoso 2013; Jurmain y Villotte 2010; Mariotti et al. 2004, 2007; Santos et al. 2010; Villotte 2008; Villotte et al. 2010; Villotte y Knüsel 2013; Weiss 2007), es muy reciente que se comienza a dar una aproximación hacia un consenso (Henderson y Alves-Cardoso 2013; Villotte y Knüsel 2013).

La información sobre EAD fue totalizada por área anatómica: hombro, codo, muñeca, mano, articulación coxofemoral, rodilla, tobillo, pie (Larsen et al. 1995, según la Tabla 2 de Klaus et al. 2009), la articulación temporomandibular (Jurmain 1990) y las costillas. Si alguna de las manifestaciones consideradas (exceptuando la porosidad aislada) estaba presente en la articulación observada, la EAD fue registrada como positiva para toda la articulación (Rojas-Sepúlveda et al. 2008), y se asignó el código más alto.

Tabla 3. Sitios de origen e inserción para observación de CE. Observed sites for EC.

\begin{tabular}{cc}
\hline Elemento óseo & Sitios observados \\
\hline Clavículas & $\begin{array}{c}\text { Origen del músculo pectoral mayor } \\
\text { Inserción del músculo trapecio } \\
\text { Fijación del ligamento costoclavicular }\end{array}$ \\
\hline \multirow{2}{*}{ Húmeros } & $\begin{array}{c}\text { Inserción del músculo pectoral mayor } \\
\text { Inserción del músculo gran dorsal } \\
\text { Inserción del músculo deltoides }\end{array}$ \\
\hline Ulnas & Inserción del músculo tríceps braquial \\
Radios & Inserción del músculo braquial \\
\hline \multirow{2}{*}{ Fémures } & Inserción del músculo bíceps braquial (fibrocartilaginosa) \\
\hline Tibias & Inserción del músculo glúteo máximo \\
& Origen del músculo vasto lateral \\
& Origen del músculo vasto medial \\
& Origen del músculo gastrocnemio \\
\hline
\end{tabular}


Las frecuencias o prevalencias de EAD y de CE fueron calculadas teniendo en cuenta solo el número de individuos que tuvieran la articulación o el sitio (de inserción u origen) observable (Waldron 1994, 2009). El numerador " $n$ " correspondió al número de individuos registrados como positivos para el elemento anatómico (articulación o sitio de inserción u origen), y el denominador "N" correspondió al número de individuos para los cuales el elemento anatómico era observable (Rojas-Sepúlveda 2009; Rojas-Sepúlveda et al. 2008).

Las frecuencias de CE fueron calculadas para cada sitio (de inserción u origen). Si bien al recolectar la información se asignaron códigos de 0 a 6 con respecto a los CE, siguiendo la metodología de Hawkey y Merbs (1995), algunos resultados preliminares mostraron que las frecuencias obtenidas al considerar los códigos 1 y 2 incluían casi la totalidad de la muestra, lo cual no permitía ver diferencias entre grupos (Rojas-Sepúlveda 2009); por dicha razón, los análisis contemplan sólo los códigos 3, 4, 5 y 6 .
Con el fin de establecer las diferencias entre los grupos, se aplicó el test de Chi cuadrado. Para valores esperados menores a 5 se utilizó el test exacto de Fisher.

Se realizó un análisis por clasificación jerárquica con el fin de ordenar las colecciones en grupos de acuerdo con las frecuencias de EAD y de $\mathrm{CE}$, teniendo en cuenta ambos indicadores, luego solamente la EAD y al final, los CE. Esto permitió observar la similitud entre las colecciones que conformaron un mismo grupo. Para estos análisis por clasificación jerárquica se aplicó el método de Ward y las distancias euclidianas. Las estadísticas se realizaron utilizando el software R 2.8.1.

\section{Resultados}

La composición de las colecciones estudiadas se presenta en la Figura 2. Nótese la baja proporción de individuos de más de 45 años.

Las Tablas 4 y 5 presentan las frecuencias de EAD y de CE. Se observa una gran variabilidad en

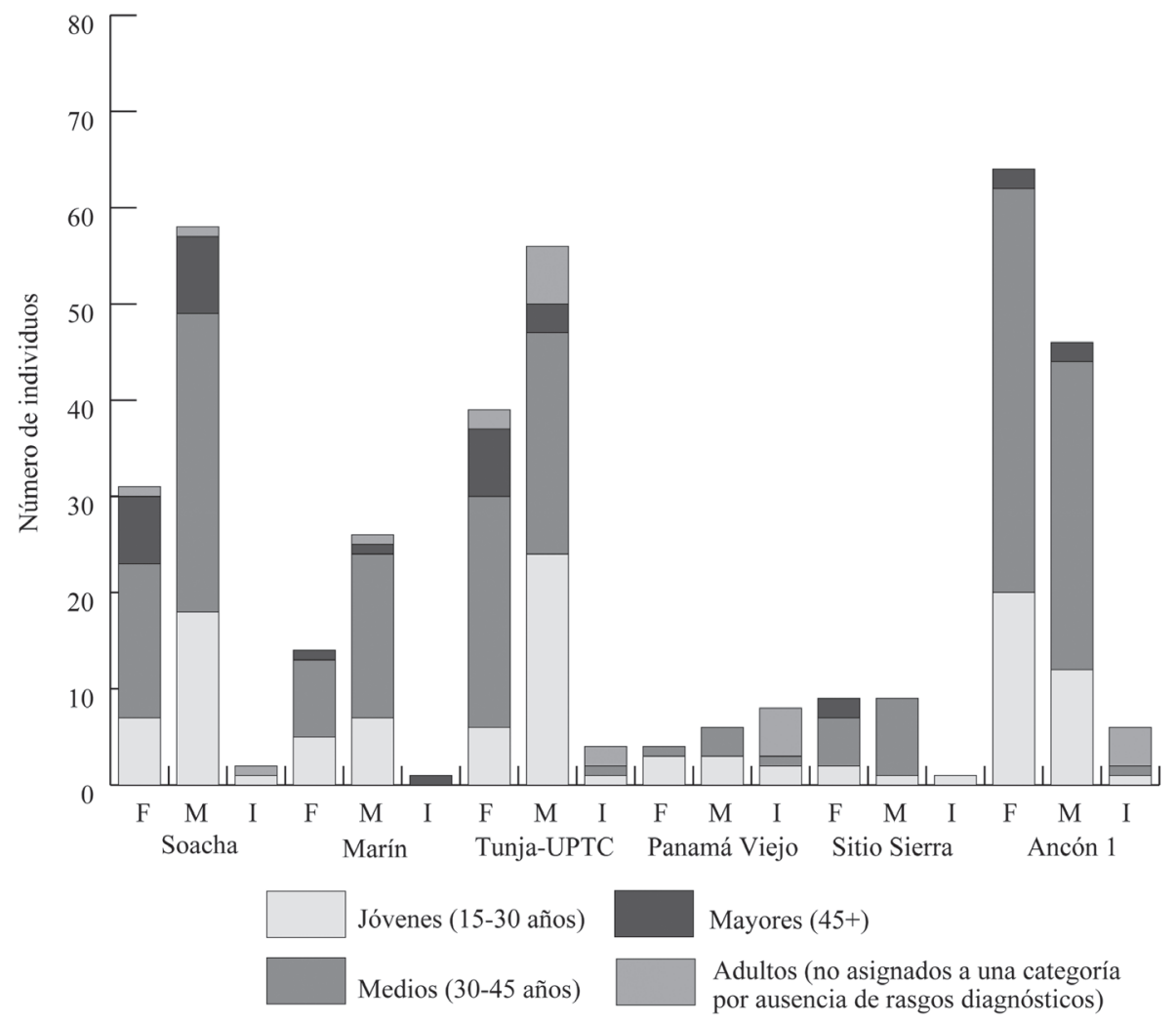

Figura 2. Composición de las muestras por edad y sexo.

Age and sex composition of the samples. 
Tabla 4. Prevalencia de la EAD por lado. DJD prevalence by side.

\begin{tabular}{|c|c|c|c|c|c|c|c|c|c|c|c|c|}
\hline \multirow{3}{*}{ Sistema articular } & \multicolumn{4}{|c|}{ Soacha } & \multicolumn{4}{|c|}{ Marín } & \multicolumn{4}{|c|}{ Tunja-UPTC } \\
\hline & \multicolumn{2}{|c|}{ Izq. } & \multicolumn{2}{|c|}{ Der. } & \multicolumn{2}{|c|}{ Izq. } & \multicolumn{2}{|c|}{ Der. } & \multicolumn{2}{|c|}{ Izq. } & \multicolumn{2}{|c|}{ Der. } \\
\hline & $\mathrm{n} / \mathrm{N}$ & $\%$ & $\mathrm{n} / \mathrm{N}$ & $\%$ & $\mathrm{n} / \mathrm{N}$ & $\%$ & $\mathrm{n} / \mathrm{N}$ & $\%$ & $\mathrm{n} / \mathrm{N}$ & $\%$ & $\mathrm{n} / \mathrm{N}$ & $\%$ \\
\hline Temporomandibular & $8 / 75$ & 10,67 & $7 / 78$ & 8,97 & $15 / 37$ & 40,54 & $8 / 37$ & 21,62 & $6 / 46$ & 13,04 & $5 / 50$ & 10,00 \\
\hline Esternoclavicular & $3 / 42$ & 7,14 & $3 / 40$ & 7,50 & $2 / 23$ & 8,70 & $4 / 24$ & 16,67 & $1 / 40$ & 2,50 & $1 / 42$ & 2,38 \\
\hline Hombro & $8 / 68$ & 11,76 & $7 / 68$ & 10,29 & $1 / 36$ & 2,78 & $1 / 37$ & 2,70 & $11 / 57$ & 19,30 & $15 / 61$ & 24,59 \\
\hline Codo & $11 / 62$ & 17,74 & $9 / 64$ & 14,06 & $7 / 38$ & 18,42 & $9 / 35$ & 25,71 & $9 / 66$ & 13,64 & $13 / 68$ & 19,12 \\
\hline Muñeca & $5 / 50$ & 10,00 & $6 / 51$ & 11,76 & $7 / 37$ & 18,92 & $6 / 32$ & 18,75 & $3 / 54$ & 5,56 & $5 / 54$ & 9,26 \\
\hline Mano & $9 / 36$ & 25,00 & $7 / 37$ & 18,92 & $6 / 27$ & 22,22 & $9 / 28$ & 32,14 & $1 / 22$ & 4,55 & $6 / 32$ & 18,75 \\
\hline Cadera & $18 / 71$ & 25,35 & $19 / 75$ & 25,33 & $3 / 33$ & 9,09 & $3 / 34$ & 8,82 & $5 / 65$ & 7,69 & $4 / 67$ & 5,97 \\
\hline Rodilla & $4 / 66$ & 6,06 & $7 / 69$ & 10,14 & $3 / 36$ & 8,33 & $3 / 36$ & 8,33 & $4 / 53$ & 7,55 & $10 / 59$ & 16,95 \\
\hline Tibiofibular & $0 / 23$ & 0,00 & $0 / 27$ & 0,00 & $0 / 25$ & 0,00 & $2 / 23$ & 8,70 & $2 / 30$ & 6,67 & $0 / 29$ & 0,00 \\
\hline Tobillo & $4 / 67$ & 5,97 & $3 / 66$ & 4,55 & $6 / 34$ & 17,65 & $5 / 34$ & 14,71 & $3 / 65$ & 4,62 & $3 / 65$ & 4,62 \\
\hline Pie & $15 / 40$ & 37,50 & $18 / 37$ & 48,65 & $16 / 31$ & 51,61 & $15 / 29$ & 51,72 & $7 / 22$ & 31,82 & $8 / 28$ & 28,57 \\
\hline \multirow{3}{*}{ Sistema articular } & \multicolumn{4}{|c|}{ Panamá Viejo } & \multicolumn{4}{|c|}{ Sitio Sierra } & \multicolumn{4}{|c|}{ Ancón 1} \\
\hline & \multicolumn{2}{|c|}{ Izq. } & \multicolumn{2}{|c|}{ Der. } & \multicolumn{2}{|c|}{ Izq. } & \multicolumn{2}{|c|}{ Der. } & \multicolumn{2}{|c|}{ Izq. } & \multicolumn{2}{|c|}{ Der. } \\
\hline & $\mathrm{n} / \mathrm{N}$ & $\%$ & $\mathrm{n} / \mathrm{N}$ & $\%$ & $\mathrm{n} / \mathrm{N}$ & $\%$ & $\mathrm{n} / \mathrm{N}$ & $\%$ & $\mathrm{n} / \mathrm{N}$ & $\%$ & $\mathrm{n} / \mathrm{N}$ & $\%$ \\
\hline Temporomandibular & $0 / 9$ & 0,00 & $0 / 8$ & 0,00 & $1 / 9$ & 11,11 & $2 / 10$ & 20,00 & $4 / 93$ & 4,30 & $4 / 93$ & 4,30 \\
\hline Esternoclavicular & $0 / 2$ & 0,00 & $0 / 3$ & 0,00 & $1 / 5$ & 20,00 & $0 / 4$ & 0,00 & $9 / 74$ & 12,16 & $10 / 75$ & 13,33 \\
\hline Hombro & $1 / 8$ & 12,50 & $3 / 6$ & 50,00 & $3 / 11$ & 27,27 & $3 / 10$ & 30,00 & $45 / 95$ & 47,37 & $50 / 97$ & 51,55 \\
\hline Codo & $1 / 11$ & 9,09 & $2 / 9$ & 22,22 & $3 / 12$ & 25,00 & $4 / 12$ & 33,33 & $35 / 100$ & 35,00 & $47 / 101$ & 46,53 \\
\hline Muñeca & $0 / 5$ & 0,00 & $0 / 4$ & 0,00 & $0 / 7$ & 0,00 & $1 / 6$ & 16,67 & $27 / 92$ & 29,35 & $26 / 77$ & 33,77 \\
\hline Mano & $2 / 4$ & 50,00 & $2 / 4$ & 50,00 & $2 / 5$ & 40,00 & $4 / 9$ & 44,44 & $15 / 38$ & 39,47 & $15 / 41$ & 36,59 \\
\hline Cadera & $1 / 8$ & 12,50 & $1 / 8$ & 12,50 & $2 / 11$ & 18,18 & $3 / 10$ & 30,00 & $40 / 101$ & 39,60 & $38 / 101$ & 37,62 \\
\hline Rodilla & $2 / 5$ & 40,00 & $1 / 5$ & 20,00 & $3 / 15$ & 20,00 & $2 / 14$ & 14,29 & $53 / 102$ & 51,96 & $50 / 104$ & 48,08 \\
\hline Tibiofibular & $0 / 1$ & 0,00 & $0 / 2$ & 0,00 & $0 / 2$ & 0,00 & $0 / 5$ & 0,00 & $7 / 96$ & 7,29 & $6 / 98$ & 6,12 \\
\hline Tobillo & $2 / 5$ & 40,0 & $2 / 6$ & 33,33 & $0 / 11$ & 0,00 & $1 / 11$ & 9,09 & $22 / 98$ & 22,45 & $23 / 102$ & 22,55 \\
\hline Pie & $2 / 5$ & 40,0 & $1 / 3$ & 33,33 & $4 / 9$ & 44,44 & $4 / 8$ & 50,00 & $34 / 43$ & 79,07 & $32 / 44$ & 72,73 \\
\hline
\end{tabular}

Izq. lado izquierdo; Der. lado derecho; n: número de casos positivos, N: número de elementos observables.

las frecuencias y perfiles de EAD y de CE en las colecciones estudiadas. Los individuos de Ancón 1 en la costa peruana presentan las frecuencias más altas de EAD en ocho de las 11 áreas articulares observadas. Los individuos de las colecciones muiscas del altiplano colombiano (Marín, Soacha y Tunja-UPTC) presentan las frecuencias más altas de CE. En el caso de la colección Marín, siete de los 14 sitios de inserción u origen observados presentan las frecuencias más elevadas. Como se puede observar en las Tablas 6 y 7, son múltiples las diferencias altamente significativas entre las frecuencias de los indicadores en las colecciones estudiadas. En general, a partir de los resultados, para $\mathrm{EAD}$, las diferencias entre las frecuencias son significativas cuando las colecciones muiscas (Soacha, Marín y Tunja-UPTC) se comparan entre sí, pero en particular cuando estas se comparan con la colección Ancón 1 (Tabla 6). En el caso de los
$\mathrm{CE}$, las diferencias son altamente significativas cuando se comparan las frecuencias al interior de las colecciones muiscas y también cuando estas se comparan con la colección de Ancón 1 (Tabla 7).

Los árboles muestran que las colecciones muiscas se ubican juntas tanto cuando se incluyen en el análisis las frecuencias de la EAD, como cuando se incluyen las frecuencias de los CE, como cuando se incluyen las frecuencias de la EAD y de los CE juntas (Figura 3).

\section{Discusión}

Estos resultados muestran una importante variabilidad entre las frecuencias y los perfiles de la EAD y de los CE en las seis colecciones óseas estudiadas. Sin embargo, hay similitudes entre las colecciones que vienen de ambientes parecidos y que presentaban sistemas de explotación similares. 
Tabla 5. Prevalencia de los CE por lado. EC prevalence by side.

\begin{tabular}{|c|c|c|c|c|c|c|c|c|c|c|c|c|}
\hline \multirow{3}{*}{ Sitio ins/orig } & \multicolumn{4}{|c|}{ Soacha } & \multicolumn{4}{|c|}{ Marín } & \multicolumn{4}{|c|}{ Tunja-UPTC } \\
\hline & \multicolumn{2}{|c|}{ Izq. } & \multicolumn{2}{|c|}{ Der. } & \multicolumn{2}{|c|}{ Izq. } & \multicolumn{2}{|c|}{ Der. } & \multicolumn{2}{|c|}{ Izq. } & \multicolumn{2}{|c|}{ Der. } \\
\hline & $\mathrm{n} / \mathrm{N}$ & $\%$ & $\mathrm{n} / \mathrm{N}$ & $\%$ & $\mathrm{n} / \mathrm{N}$ & $\%$ & $\mathrm{n} / \mathrm{N}$ & $\%$ & $\mathrm{n} / \mathrm{N}$ & $\%$ & $\mathrm{n} / \mathrm{N}$ & $\%$ \\
\hline C. pectoral mayor & $2 / 54$ & 3,7 & $5 / 54$ & 9,26 & $0 / 26$ & 0,00 & $2 / 30$ & 6,67 & $1 / 42$ & 2,38 & $0 / 53$ & 0,00 \\
\hline C. costoclavicular & $21 / 57$ & 36,84 & $28 / 57$ & 49,12 & $10 / 28$ & 35,71 & $14 / 31$ & 45,16 & $18 / 54$ & 33,33 & $23 / 56$ & 41,07 \\
\hline C. trapecio & $14 / 65$ & 21,54 & $15 / 67$ & 22,39 & $13 / 33$ & 39,39 & $9 / 32$ & 28,13 & $1 / 60$ & 1,67 & $1 / 55$ & 1,82 \\
\hline H. pectoral mayor & $39 / 73$ & 53,42 & $37 / 75$ & 45,33 & $22 / 41$ & 53,66 & $28 / 39$ & 71,79 & $33 / 73$ & 45,21 & $34 / 3$ & 46,58 \\
\hline H. gran dorsal & $5 / 71$ & 7,04 & $7 / 69$ & 10,14 & $8 / 29$ & 27,59 & $8 / 29$ & 27,59 & $10 / 64$ & 15,63 & $8 / 59$ & 13,56 \\
\hline H. deltoides & $13 / 74$ & 17,57 & $14 / 77$ & 18,18 & $11 / 39$ & 28,21 & $14 / 39$ & 35,9 & $20 / 74$ & 27,03 & $22 / 74$ & 29,73 \\
\hline R. bíceps braquial & $2 / 63$ & 3,17 & $1 / 62$ & 1,61 & $12 / 34$ & 35,29 & $18 / 37$ & 48,65 & $22 / 70$ & 31,43 & $21 / 72$ & 29,17 \\
\hline U. tríceps braquial & $0 / 35$ & 0,00 & $0 / 38$ & 0,00 & $1 / 28$ & 3,57 & $0 / 33$ & 0,00 & $1 / 59$ & 1,69 & $1 / 59$ & 1,69 \\
\hline U. braquial & $3 / 63$ & 4,76 & $3 / 66$ & 4,55 & $2 / 33$ & 6,06 & $2 / 38$ & 5,26 & $4 / 76$ & 5,26 & $5 / 74$ & 6,76 \\
\hline F. glúteo máximo & $34 / 67$ & 50,75 & $30 / 67$ & 44,78 & $28 / 39$ & 71,79 & $26 / 40$ & 65 & $28 / 74$ & 37,84 & $27 / 72$ & 37,5 \\
\hline F. vasto medial & $10 / 76$ & 13,16 & $6 / 79$ & 7,59 & $11 / 41$ & 26,83 & $10 / 41$ & 24,39 & $13 / 78$ & 16,67 & $13 / 76$ & 17,11 \\
\hline F. vasto lateral & $12 / 77$ & 15,58 & $13 / 80$ & 16,25 & $12 / 40$ & 30 & $11 / 40$ & 27,5 & $9 / 77$ & 11,69 & $7 / 76$ & 9,21 \\
\hline F. gastrocnemio & $7 / 56$ & 12,5 & $9 / 61$ & 14,75 & $0 / 17$ & 0,00 & $0 / 14$ & 0,00 & $20 / 54$ & 37,04 & $16 / 53$ & 30,19 \\
\hline T. sóleo & $11 / 67$ & 16,42 & $11 / 64$ & 17,19 & $7 / 39$ & 17,95 & $6 / 37$ & 16,22 & $11 / 67$ & 16,42 & $9 / 68$ & 13,24 \\
\hline \multirow{3}{*}{ Sitio ins/orig } & \multicolumn{4}{|c|}{ Panamá Viejo } & \multicolumn{4}{|c|}{ Sitio Sierra } & \multicolumn{4}{|c|}{ Ancón 1} \\
\hline & \multicolumn{2}{|c|}{ Izq. } & \multicolumn{2}{|c|}{ Der. } & \multicolumn{2}{|c|}{ Izq. } & \multicolumn{2}{|c|}{ Der. } & \multicolumn{2}{|c|}{ Izq. } & \multicolumn{2}{|c|}{ Der. } \\
\hline & $\mathrm{n} / \mathrm{N}$ & $\%$ & $\mathrm{n} / \mathrm{N}$ & $\%$ & $\mathrm{n} / \mathrm{N}$ & $\%$ & $\mathrm{n} / \mathrm{N}$ & $\%$ & $\mathrm{n} / \mathrm{N}$ & $\%$ & $\mathrm{n} / \mathrm{N}$ & $\%$ \\
\hline C. pectoral mayor & $0 / 8$ & 0,00 & $0 / 5$ & 0,00 & $1 / 10$ & 10,00 & $0 / 8$ & 0,00 & $3 / 43$ & 6,98 & $1 / 44$ & 2,27 \\
\hline C. costoclavicular & $1 / 3$ & 33,33 & $2 / 3$ & 66,67 & $2 / 4$ & 50,00 & $4 / 4$ & 100,00 & $45 / 91$ & 49,45 & $48 / 91$ & 52,75 \\
\hline C. trapecio & $1 / 8$ & 12,5 & $2 / 9$ & 22,22 & $1 / 12$ & 8,33 & $0 / 12$ & 0,00 & $7 / 94$ & 7,45 & $2 / 92$ & 2,17 \\
\hline H. pectoral mayor & $3 / 12$ & 25,00 & $1 / 13$ & 7,69 & $1 / 13$ & 7,69 & $2 / 15$ & 13,33 & $18 / 101$ & 17,82 & $12 / 102$ & 11,76 \\
\hline H. gran dorsal & $0 / 1$ & 0,00 & $0 / 2$ & 0,00 & $0 / 0$ & 0,00 & $0 / 0$ & 0,00 & $4 / 50$ & 8,00 & $2 / 51$ & 3,92 \\
\hline H. deltoides & $3 / 13$ & 23,08 & $2 / 11$ & 18,18 & $2 / 14$ & 14,29 & $1 / 15$ & 6,67 & $37 / 101$ & 36,63 & $42 / 102$ & 41,18 \\
\hline R. bíceps braquial & $3 / 8$ & 37,5 & $4 / 8$ & 50,00 & $2 / 12$ & 16,67 & $2 / 13$ & 15,38 & $26 / 95$ & 27,37 & $29 / 96$ & 30,21 \\
\hline U. tríceps braquial & $0 / 9$ & 0,00 & $0 / 9$ & 0,00 & $0 / 10$ & 0,00 & $0 / 10$ & 0,00 & $0 / 78$ & 0,00 & $0 / 81$ & 0,00 \\
\hline U. braquial & $0 / 11$ & 0,00 & $0 / 10$ & 0,00 & $0 / 13$ & 0,00 & $2 / 16$ & 12,5 & $3 / 97$ & 3,09 & $4 / 97$ & 4,12 \\
\hline F. glúteo máximo & $3 / 8$ & 37,5 & $2 / 9$ & 22,22 & $6 / 15$ & 40,00 & $5 / 13$ & 38,46 & $52 / 106$ & 49,06 & $49 / 108$ & 45,37 \\
\hline F. vasto medial & $1 / 10$ & 10,00 & $1 / 10$ & 10,00 & $1 / 16$ & 6,25 & $0 / 17$ & 0,00 & $21 / 108$ & 19,44 & $22 / 107$ & 20,56 \\
\hline F. vasto lateral & $1 / 10$ & 10,00 & $0 / 10$ & 0,00 & $1 / 16$ & 6,25 & $0 / 17$ & 0,00 & $19 / 108$ & 17,59 & $20 / 107$ & 18,69 \\
\hline F. gastrocnemio & $0 / 2$ & 0,00 & $0 / 1$ & 0,00 & $0 / 2$ & 0,00 & $0 / 5$ & 0,00 & $7 / 95$ & 7,37 & 9/97 & 9,28 \\
\hline T. sóleo & $1 / 7$ & 14,29 & $2 / 8$ & 25 & $1 / 11$ & 9,09 & $1 / 13$ & 7,69 & $22 / 103$ & 21,36 & $23 / 103$ & 22,33 \\
\hline
\end{tabular}

Izq. lado izquierdo; Der. lado derecho; n: número de casos positivos, N: número de elementos observables; C: clavícula; H: húmero; R: radio; U: ulna; F: fémur; T: tibia.

La variabilidad tanto en las frecuencias como en los perfiles de EAD ha sido expuesta como argumento para apoyar la relación entre la actividad física y la EAD (Bridges 1992). Ambos indicadores tienden a estar más presentes en el lado derecho que en el izquierdo, lo cual también ha ayudado a probar una relación positiva entre la EAD y la actividad física (Jurmain 1977, 1980; Ortner 1968), argumentos que podrían equipararse para los CE.

En los resultados obtenidos se observa una falta de concordancia entre las frecuencias de la EAD y de los CE. Las colecciones en las cuales se presentaron las frecuencias más altas de EAD no presentaron las frecuencias más altas de CE. Sin embargo, el análisis de clasificación jerárquica formó grupos de las colecciones provenientes de ambientes similares, lo cual permitió comprender que los resultados eran coherentes y que podrían ser explicados.

Si bien ambos indicadores se relacionan con múltiples factores, vale la pena reflexionar en forma breve sobre el papel del que ha sido considerado uno de los más importantes. La edad suele relacionarse con la EAD (Bourke 1967; Bridges 1992; Jurmain 1977, 1980; Jurmain y Kilgore 1995; Molnar et al. 2011), y con los CE (Cardoso y Henderson 2010; 
Tabla 6. Resultados de Chi cuadrado, comparación de la frecuencia de la EAD en los miembros superiores e inferiores (diferencias altamente significativas).

Chi square results, comparisons of DJD prevalence in upper and lower body (highly significant differences).

\begin{tabular}{|c|c|c|}
\hline Colecciones comparadas & EAD en miembros superiores & EAD en miembros inferiores \\
\hline Soacha/Marín & Temporomandibular I $\left(\chi^{2}=13,550 ; P=0,000\right)$ & Cadera $\mathrm{D}\left(\chi^{2}=3,958 ; P=0,047\right)$ \\
\hline Soacha/Tunja-UPTC & Hombro D $\left(\chi^{2}=4,646 ; P=0,031\right)$ & $\begin{array}{l}\text { Cadera I }\left(\chi^{2}=7,531 ; P=0,006\right) \\
\text { Cadera } \mathrm{D}\left(\chi^{2}=9,775 ; P=0,002\right)\end{array}$ \\
\hline Soacha/Panamá Viejo & Hombro D $(P=0,029)$ & \\
\hline Soacha/Ancón 1 & $\begin{array}{c}\text { Hombro I }\left(\chi^{2}=22,895 ; P=0,000\right) \\
\text { Hombro D }\left(\chi^{2}=30,086 ; P=0,000\right) \\
\text { Codo I }\left(\chi^{2}=5,606 ; P=0,018\right) \\
\text { Codo D }\left(\chi^{2}=18,424 ; P=0,000\right) \\
\text { Muñeca I }\left(\chi^{2}=6,947 ; P=0,008\right) \\
\text { Muñeca D }\left(\chi^{2}=7,921 ; P=0,005\right)\end{array}$ & $\begin{array}{c}\text { Rodilla I }\left(\chi^{2}=37,660 ; P=0,000\right) \\
\text { Rodilla D }\left(\chi^{2}=27,015 ; P=0,000\right) \\
\text { Tobillo I }\left(\chi^{2}=8,141 ; P=0,004\right) \\
\text { Tobillo D }\left(\chi^{2}=9,929 ; P=0,002\right) \\
\text { Pie I }\left(\chi^{2}=14,808 ; P=0,000\right) \\
\text { Pie D }\left(\chi^{2}=4,933 ; P=0,026\right)\end{array}$ \\
\hline Marín/Tunja-UPTC & $\begin{array}{c}\text { Temporomandibular I }\left(\chi^{2}=8,204 ; P=0,004\right) \\
\text { Hombro I }(P=0,025) \\
\text { Hombro D }\left(\chi^{2}=8,076 ; P=0,004\right)\end{array}$ & \\
\hline Marín/Panamá Viejo & $\begin{array}{c}\text { Temporomandibular I }(P=0,021) \\
\text { Hombro } \mathrm{D}(P=0,006)\end{array}$ & \\
\hline Marín/Sitio Sierra & $\begin{array}{l}\text { Hombro I }(P=0,035) \\
\text { Hombro D }(P=0,026)\end{array}$ & \\
\hline Marín/Ancón 1 & $\begin{array}{c}\text { Temporomandibular I }\left(\chi^{2}=27,856 ; P=0,000\right) \\
\text { Temporomandibular } \mathrm{D}(P=0,005) \\
\text { Hombro I }\left(\chi^{2}=22,783 ; P=0,000\right) \\
\text { Hombro } \mathrm{D}\left(\chi^{2}=27,105 ; P=0,000\right) \\
\text { Codo D }\left(\chi^{2}=4,652 ; P=0,031\right)\end{array}$ & $\begin{array}{c}\text { Cadera I }\left(\chi^{2}=10,627 ; P=0,001\right) \\
\text { Cadera D }\left(\chi^{2}=9,977 ; P=0,002\right) \\
\text { Rodilla I }\left(\chi^{2}=21,004 ; P=0,000\right) \\
\text { Rodilla D }\left(\chi^{2}=17,956 ; P=0,000\right) \\
\quad \text { Pie I }\left(\chi^{2}=6,197 ; P=0,013\right)\end{array}$ \\
\hline Tunja-UPTC/Panamá Viejo & & Tobillo I $(P=0,038)$ \\
\hline Tunja-UPTC/Sitio Sierra & & Cadera D $(P=0,043)$ \\
\hline Tunja-UPTC/Ancón 1 & $\begin{array}{c}\text { Hombro I }\left(\chi^{2}=12,064 ; P=0,001\right) \\
\text { Hombro D }\left(\chi^{2}=11,238 ; P=0,001\right) \\
\text { Codo I }\left(\chi^{2}=9,315 ; P=0,002\right) \\
\text { Codo D }\left(\chi^{2}=13,341 ; P=0,000\right) \\
\text { Muñeca I }\left(\chi^{2}=11,799 ; P=0,001\right) \\
\text { Muñeca D }\left(\chi^{2}=10,553 ; P=0,001\right) \\
\text { Mano I }\left(\chi^{2}=8,692 ; P=0,003\right)\end{array}$ & $\begin{array}{c}\text { Cadera I }\left(\chi^{2}=20,382 ; P=0,000\right) \\
\text { Cadera D }\left(\chi^{2}=21,525 ; P=0,000\right) \\
\text { Rodilla I }\left(\chi^{2}=29,590 ; P=0,000\right) \\
\text { Rodilla D }\left(\chi^{2}=15,681 ; P=0,000\right) \\
\text { Tobillo I }\left(\chi^{2}=9,572 ; P=0,002\right) \\
\text { Tobillo D }\left(\chi^{2}=9,714 ; P=0,002\right) \\
\text { Pie I }\left(\chi^{2}=13,952 ; P=0,000\right) \\
\text { Pie D }\left(\chi^{2}=13,512 ; P=0,000\right)\end{array}$ \\
\hline Sitio Sierra/Ancón 1 & & $\begin{array}{l}\text { Rodilla I }(P=0,026) \\
\text { Rodilla D }(P=0,021) \\
\quad \text { Pie I }(P=0,048)\end{array}$ \\
\hline
\end{tabular}

I: izquierdo, D: derecho. Fisher en cursiva.

Kennedy 1998; Mariotti et al. 2004; Molnar 2006; Molnar et al. 2011; Niinimäki 2011; Robb 1998; Stirland 1998; Villotte 2008; Villotte et al. 2010; Weiss 2003b, 2004, 2007; Weiss et al. 2012; Wilczak 1998), pero en este estudio la edad no sesga los resultados, ya que las colecciones no presentan números significativos de individuos de más de 45 años. Se ha encontrado que los CE se correlacionan de manera significativa con la edad cuando el nivel de estrés físico del grupo es bajo, pero la correlación es mucho menos clara cuando el nivel de estrés físico es alto (Havelková et al. 2011); se han reportado diferencias entre las frecuencias de grupos de individuos de 20 a 50 años, pero no entre grupos de individuos de más de 50 años (Havelkonvà et al. 2011), es decir, que antes de los 50 años, estos indicadores muestran claramente la influencia de la actividad física. Un estudio realizado sobre esqueletos de edad, sexo y ocupación conocidos muestra que las diferencias de las prevalencias de $\mathrm{CE}$ entre grupos con diferentes actividades observadas en el lado derecho y en el lado izquierdo son más importantes antes de los 50 años (Villotte et al. 2010).

Por otro lado, se evitaron sesgos al excluir del estudio individuos que presentaban trauma significativo, malformaciones congénitas, DISH (Diffuse idiopathic skeletal hiperostosis) o espondilitis anquilosante. 
Tabla 7. Resultados Chi cuadrado, comparaciones de la frecuencia de CE, miembros superiores e inferiores (diferencias altamente significativas).

Chi square results, comparisons of EC prevalence in upper and lower body (highly significant differences).

\begin{tabular}{|c|c|c|}
\hline Col. Comp. & CE $3+4+5+6$ en miembros superiores & CE $3+4+5+6$ en miembros inferiores \\
\hline $\begin{array}{l}\text { Soacha/ } \\
\text { Marín }\end{array}$ & $\begin{array}{l}\text { H. pectoral mayor } \mathrm{D}\left(\chi^{2}=7,242 ; P=0,007\right) \\
\text { H. gran dorsal } \mathrm{I}(P=0,009) \\
\text { H. deltoides } \mathrm{D}\left(\chi^{2}=4,437 ; P=0,035\right) \\
\text { R. bíceps braquial } \mathrm{I}(P=0,000) \\
\text { R. bíceps braquial } \mathrm{D}\left(\chi^{2}=33,055 ; P=0,000\right)\end{array}$ & $\begin{array}{l}\text { F. glúteo máximo I }\left(\chi^{2}=4,498 ; P=0,034\right) \\
\text { F. glúteo máximo } \mathrm{D}\left(\chi^{2}=4,107 ; P=0,043\right) \\
\text { F. vasto medial } \mathrm{D}\left(\chi^{2}=6,589 ; P=0,010\right)\end{array}$ \\
\hline $\begin{array}{c}\text { Soacha/ } \\
\text { Tunja-UPTC }\end{array}$ & $\begin{array}{l}\text { C. trapecio I }\left(\chi^{2}=11,667 ; P=0,001\right) \\
\text { C. trapecio } \mathrm{D}\left(\chi^{2}=11,216 ; P=0,001\right) \\
\text { R. bíceps braquial I }\left(\chi^{2}=17,898 ; P=0,000\right) \\
\text { R. bíceps braquial } \mathrm{D}\left(\chi^{2}=18,431 ; P=0,000\right)\end{array}$ & $\begin{array}{l}\left.\text { F. gastrocnemio I ( } \chi^{2}=8,937 ; P=0,003\right) \\
\text { F. gastrocnemio } \mathrm{D}\left(\chi^{2}=3,946 ; P=0,047\right)\end{array}$ \\
\hline $\begin{array}{c}\text { Soacha/ } \\
\text { Panamá Viejo }\end{array}$ & $\begin{array}{c}\text { H. pectoral mayor } \mathrm{D}\left(\chi^{2}=6,553 ; P=0,010\right) \\
\text { R. bíceps braquial } \mathrm{I}(P=0,009) \\
\text { R. bíceps braquial } \mathrm{D}(P=0,000)\end{array}$ & \\
\hline $\begin{array}{l}\text { Soacha/ } \\
\text { Sitio Sierra }\end{array}$ & $\begin{array}{l}\text { H. pectoral mayor I }\left(\chi^{2}=9,277 ; P=0,002\right) \\
\text { H. pectoral mayor } \mathrm{D}\left(\chi^{2}=5,333 ; P=0,021\right)\end{array}$ & \\
\hline $\begin{array}{l}\text { Soacha/ } \\
\text { Ancón } 1\end{array}$ & $\begin{array}{c}\text { C. trapecio I }\left(\chi^{2}=6,657 ; P=0,010\right) \\
\text { C. trapecio } \mathrm{D}\left(\chi^{2}=16,590 ; P=0,000\right) \\
\text { H. pectoral mayor I }\left(\chi^{2}=24,384 ; P=0,000\right) \\
\text { H. pectoral mayor } \mathrm{D}\left(\chi^{2}=25,321 ; P=0,000\right) \\
\text { H. deltoides } \mathrm{I}\left(\chi^{2}=7,607 ; P=0,006\right) \\
\text { H. deltoides } \mathrm{D}\left(\chi^{2}=10,792 ; P=0,001\right) \\
\text { R. bíceps braquial I }\left(\chi^{2}=15,207 ; P=0,000\right) \\
\text { R. bíceps braquial } \mathrm{D}\left(\chi^{2}=20,025 ; P=0,000\right)\end{array}$ & F. vasto medial $\mathrm{D}\left(\chi^{2}=5,975 ; P=0,015\right)$ \\
\hline $\begin{array}{c}\text { Marín/ } \\
\text { Tunja-UPTC }\end{array}$ & $\begin{array}{l}\text { C. trapecio I }(P=0,000) \\
\text { C. trapecio } \mathrm{D}(P=0,000) \\
\text { H. pectoral mayor } \mathrm{D}\left(\chi^{2}=6,542 ; P=0,011\right) \\
\text { R. bíceps braquial } \mathrm{D}\left(\chi^{2}=4,037 ; P=0,045\right) \\
\text { F. gastrocnemio } \mathrm{D}(P=0,016)\end{array}$ & $\begin{array}{l}\text { F. glúteo máximo I }\left(\chi^{2}=11,781 ; P=0,001\right) \\
\text { F. glúteo máximo } \mathrm{D}\left(\chi^{2}=7,801 ; P=0,005\right) \\
\text { F. vasto lateral I }\left(\chi^{2}=5,994 ; P=0,014\right) \\
\text { F. vasto lateral } \mathrm{D}\left(\chi^{2}=6,687 ; P=0,010\right) \\
\text { F. gastrocnemio } \mathrm{I}(P=0,002)\end{array}$ \\
\hline $\begin{array}{c}\text { Marín/ } \\
\text { Panamá Viejo }\end{array}$ & H. pectoral mayor $\mathrm{D}\left(\chi^{2}=16,242 ; P=0,000\right)$ & F. glúteo máximo $\mathrm{D}(P=0,028)$ \\
\hline $\begin{array}{l}\text { Marín/ } \\
\text { Sitio Sierra }\end{array}$ & $\begin{array}{c}\text { C. trapecio } \mathrm{D}(P=0,047) \\
\text { H. pectoral mayor I }\left(\chi^{2}=8,529 ; P=0,003\right) \\
\text { H. pectoral mayor } \mathrm{D}\left(\chi^{2}=14,995 ; P=0,000\right) \\
\text { H. deltoides } \mathrm{D}(P=0,043) \\
\text { R. bíceps braquial } \mathrm{D}\left(\chi^{2}=4,435 ; P=0,035\right)\end{array}$ & $\begin{array}{l}\text { F. glúteo máximo I }\left(\chi^{2}=4,696 ; P=0,030\right) \\
\text { F. vasto medial } \mathrm{D}(P=0,026) \\
\text { F. vasto lateral } \mathrm{D}(P=0,024)\end{array}$ \\
\hline $\begin{array}{l}\text { Marín/ } \\
\text { Ancón } 1\end{array}$ & $\begin{array}{c}\text { C. trapecio I }\left(\chi^{2}=18,789 ; P=0,000\right) \\
\text { C. trapecio } \mathrm{D}(P=0,000) \\
\text { H. pectoral mayor I }\left(\chi^{2}=18,509 ; P=0,000\right) \\
\text { H. pectoral mayor } \mathrm{D}\left(\chi^{2}=50,031 ; P=0,000\right) \\
\text { H. gran dorsal } \mathrm{I}(P=0,026) \\
\text { H. gran dorsal } \mathrm{D}(P=0,004) \\
\text { R. bíceps braquial D }\left(\chi^{2}=3,974 ; P=0,046\right)\end{array}$ & $\begin{array}{l}\text { F. glúteo máximo I }\left(\chi^{2}=5,960 ; P=0,015\right) \\
\text { F. glúteo máximo } \mathrm{D}\left(\chi^{2}=4,500 ; P=0,034\right)\end{array}$ \\
\hline $\begin{array}{l}\text { Tunja-UPTC/ } \\
\text { Panamá Viejo }\end{array}$ & $\begin{array}{l}\text { C. trapecio } \mathrm{D}(P=0,050) \\
\text { H. pectoral mayor } \mathrm{D}\left(\chi^{2}=6,913 ; P=0,009\right)\end{array}$ & \\
\hline $\begin{array}{l}\text { Tunja-UPTC/ } \\
\text { Sitio Sierra }\end{array}$ & $\begin{array}{l}\text { C. costoclavicular } \mathrm{D}(P=0,036) \\
\text { H. pectoral mayor I }\left(\chi^{2}=6,496 ; P=0,011\right) \\
\text { H. pectoral mayor } \mathrm{D}\left(\chi^{2}=5,688 ; P=0,017\right)\end{array}$ & \\
\hline $\begin{array}{l}\text { Tunja-UPTC/ } \\
\text { Ancón } 1\end{array}$ & $\begin{array}{l}\text { H. pectoral mayor I }\left(\chi^{2}=15,336 ; P=0,000\right) \\
\text { H. pectoral mayor } \mathrm{D}\left(\chi^{2}=26,610 ; P=0,000\right)\end{array}$ & $\begin{array}{l}\text { F. gastrocnemio I }\left(\chi^{2}=20,426 ; P=0,000\right) \\
\text { F. gastrocnemio } \mathrm{D}\left(\chi^{2}=10,790 ; P=0,001\right)\end{array}$ \\
\hline Panamá Viejo/ Ancón 1 & C. trapecio $\mathrm{D}(P=0,039)$ & \\
\hline $\begin{array}{l}\text { Sitio Sierra/ } \\
\text { Ancón } 1\end{array}$ & H. deltoides $\mathrm{D}\left(\chi^{2}=6,700 ; P=0,010\right)$ & F. vasto medial $\mathrm{D}(P=0,041)$ \\
\hline
\end{tabular}

Col. Comp.: Colecciones comparadas. I: izquierdo, D: derecho. C: clavícula, H: húmero, R: radio, F: fémur. Fisher en cursiva. 


\section{Enfermedad articular degenerativa-EAD y Cambios entesiales-CE}

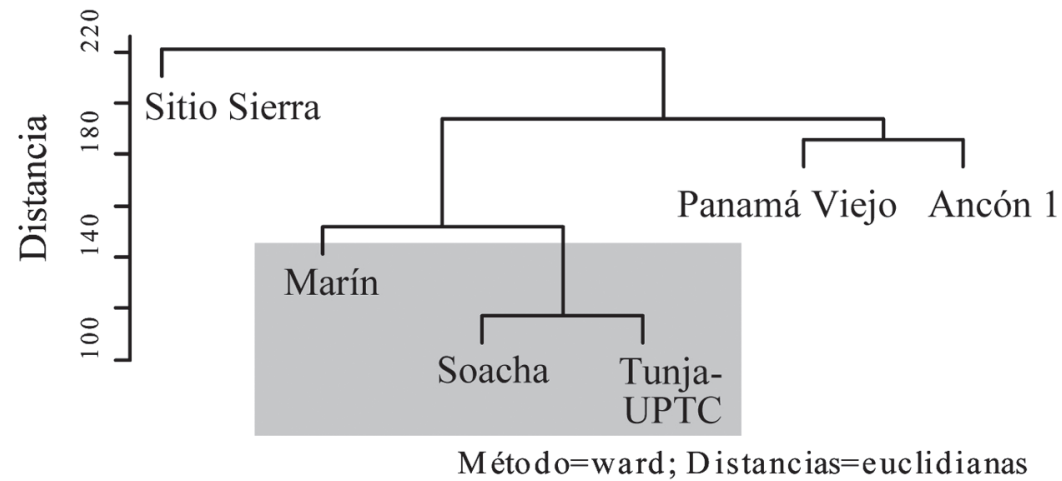

\section{Enfermedad articular Degenerativa-EAD}

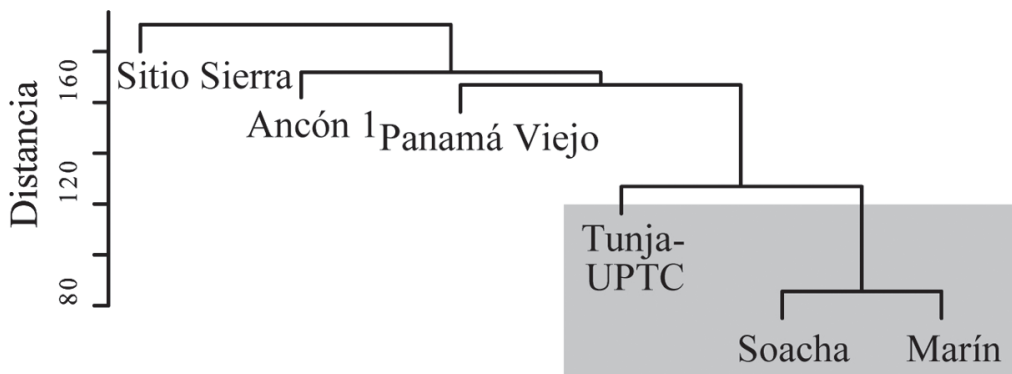

Método $=$ ward; D istancias $=$ euclidianas

\section{Cambios Entesiales-CE}

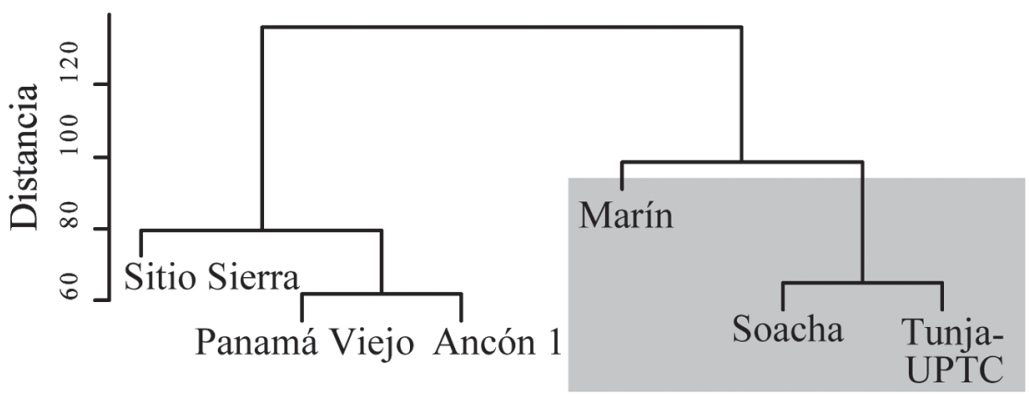

Métod $\mathrm{o}=\mathrm{w}$ ard $; \mathrm{D}$ istancias $=$ euclidianas

Figura 3. Árboles resultado de la clasificación jerárquica. 
En cuanto a la genética, su influencia es importante en términos de severidad de EAD (Weiss y Jurmain 2007), mas no sobre su mera presencia (frecuencia). La genética, sin embargo, ha sido considerada como un factor importante en la EAD sobre partes anatómicas específicas: las rodillas, las articulaciones coxofemorales y la columna vertebral (Jiménez y Dharmavaram 2009; Weiss y Jurmain 2007). Al mismo tiempo, Weiss y Jurmain (2007) consideran que en contextos prehispánicos la influencia del factor genético podría haberse visto reducida, ya que el impacto de la actividad pudo haber sido alto. Asimismo, consideran que el peso no debió haber producido efectos significativos en las frecuencias de la EAD en poblaciones premodernas (Weiss y Jurmain 2007). El peso podría influenciar especialmente la EAD en regiones anatómicas específicas, como las rodillas (Rogers y Dieppe 1994). En este estudio, las frecuencias halladas de EAD en las rodillas no son particularmente altas.

Por las razones antes expuestas, la actividad física podría tener la influencia más importante en los indicadores observados en las colecciones óseas estudiadas aquí.

Según Benjamin et al. (2006), la combinación de factores mecánicos y biomecánicos es la causa más aceptada de los cambios entesiales ("entesopatías" en el texto). La observación simultánea de estos marcadores, EAD y CE, beneficia la interpretación de los resultados. Como se ha mencionado, las colecciones con las frecuencias mayores de EAD no muestran frecuencias elevadas de CE (Ancón 1), y viceversa (Marín). A pesar de esta aparente falta de concordancia entre los indicadores, los árboles de clasificación permitieron observar que las colecciones se agrupaban de manera coherente, las colecciones muiscas de tierras altas de Colombia, con medios y sistemas de explotación de recursos análogos, formaron grupos aparte en los árboles.

A partir de los resultados obtenidos y la discusión precedente, la falta de correspondencia entre la EAD y los CE podría ser explicada por el nivel de actividad física y las cargas portadas por los individuos estudiados. De esta manera, la EAD y los CE pueden responder a diferentes tipos de actividad: actividades más repetitivas sin cargas pesadas podrían producir más EAD que $\mathrm{CE}$, cargando de manera más significativa el sistema articular que el sistema periarticular; y las actividades intensas que incluyen cargas pesadas producen hipertrofia muscular y sobrecarga de las entesis. Villotte (2008), al estudiar colecciones óseas de sexo, edad y ocupación conocidas considera que la simple repetición del movimiento no influencia de manera significativa los CE, mientras que el transporte de cargas pesadas es un factor clave. Stefanović y Porćič (2011) muestran que el tipo de actividad influencia la expresión de los CE.

Por otro lado, los CE podrían aparecer como una respuesta adaptativa a la sobrecarga del sistema musculoesquelético, actuando como protección contra la EAD. Esto es respaldado por estudios que muestran que el entrenamiento de fuerza intensa en individuos en edad prepuberal y adolescentes puede protegerlos contra las lesiones, ya que ayuda a consolidar los músculos y las articulaciones (Costill y Wilmore 2006). Además el entrenamiento físico produce un cartílago más grueso y más flexible, un aumento del área de contacto entre las articulaciones y un crecimiento en la sustancia intercelular de los tejidos conjuntivos y del volumen de los tendones y de los ligamentos (Åstrand y Rodahl 1994). Knüsel (1993) sugirió el rol "protector" de la robustez contra la EAD, dando una nueva interpretación a los resultados de Bridges (1991); sin embargo, luego de la respuesta de Bridges (1993), la hipótesis no volvió a ser formalmente discutida.

Por otro lado, teniendo en cuenta que los muiscas vivieron entre los 2.500 y 2.800 metros sobre el nivel del mar, donde la hipoxia está presente, y que las colecciones muiscas revelaron las frecuencias más elevadas de CE, se podría considerar la altitud como otro factor en los estudios de CE. Esta hipótesis no ha sido considerada en estudios bioarqueológicos previos; sin embargo, requiere mayor investigación puesto que los estudios relacionados con la hipertrofia muscular en individuos residentes a gran altura son muy escasos (Costill y Wilmore 2006; Green y Sutton 2001). Un estudio realizado con tres hombres Quechua (3.300 metros sobre el nivel del mar) demostró que las fibras musculares cambiaron su vocación, y que la adaptación a la hipoxia crónica favorece la disminución del potencial metabólico anabólico y el aumento del anaeróbico (Rosser y Hochachka 1993). Sin embargo, aún es poco claro si esto se relaciona con una adaptación genética o con una respuesta individual. 


\section{Conclusión}

Una metodología para la recolección de datos y el tratamiento de los mismos permitió la comparación directa de las frecuencias y de los perfiles de la EAD y de los CE. Una importante variabilidad de perfiles y de frecuencias fue observada, así como algunas similitudes entre colecciones óseas de medios similares y de sistemas de explotación de recursos análogos, junto con su respectivo agrupamiento en los árboles. Esto, unido a la tendencia mayor de los indicadores hacia el lado derecho, apoya la relación entre EAD, CE y actividad física. Se propone que el tipo de actividad influencia de diferentes maneras los dos marcadores estudiados: una actividad repetitiva podría tener una influencia más importante en la EAD, mientras que las actividades relacionadas con cargas pesadas y con la hipertrofia podrían influir más en los CE. La actividad física de tipo intenso realizada desde la adolescencia puede generar una respuesta adaptativa contra la EAD. Las comparaciones realizadas entre poblaciones de diferentes ecosistemas sugieren que la altitud debería ser tomada en cuenta como un nuevo factor para los estudios de CE.
Agradecimientos: Los autores agradecen a la Doctora Emma Rabino-Massa (Università di Torino); al Doctor José Vicente Rodríguez (Universidad Nacional de Colombia); al Doctor Henri Duday (CNRS-Université de Bordeaux-1); y al Doctor Jean Michel Viton (Université de la Méditerranée); por sus importantes observaciones. Asimismo, a los curadores del material estudiado, en Colombia: a Patricia Ramírez del Instituto Colombiano de Antropología e Historia; a la Profesora Helena Pradilla del Museo Arqueológico de Tunja de la Universidad Pedagógica y Tecnológica de Colombia. En Panamá: al Doctor Juan Guillermo Martín del Patronato Panamá Viejo y al Doctor Richard Cooke del Smithsonian Tropical Research Institute. En Perú: al Museo Nacional de Arqueología, Antropología e Historia del Perú-MNAAHP, especialmente a la anterior directora del Departamento de Restos Humanos, arqueóloga Elsa Tomasto. El Ministerio Francés de la Educación Superior y la Investigación financió el trabajo por medio de una beca doctoral para el primer autor. Los autores agradecen a los evaluadores anónimos del artículo, quienes le aportaron mucho con sus oportunas sugerencias.

\section{Referencias Citadas}

Acosta, M. 2012. Una mirada a los marcadores óseos de actividad: aproximación al periodo Temprano (340 a.C.-440 d.C.) del valle geográfico del río Cauca. Revista Colombiana de Antropología 48:169-187.

Álvarez, B. y J.V. Rodríguez 2001. Estudio osteométrico de los restos óseos del Cercado grande de los Santuarios, Tunja, Boyacá. En Los Chibchas: adaptación y diversidad en los Andes orientales de Colombia, editado por J.V. Rodríguez, pp. 207216. Universidad Nacional de Colombia-Colciencias, Bogotá.

Arrieta, M. y O. Mendonça 2011. Enfermedad degenerativa articular y uso del cuerpo en Rincón Chico 21 (Santa María, Catamarca). Revista Argentina de Antropología Biológica 13:3-17.

Åstrand, P.O. y K. Rodahl 1994. Précis de Physiologie de l'Exercice Musculaire. Traducido por J.-R. Lacour y D. Duizabo. Tercera edición. Masson, Paris.

Baker, O., H. Duday y O. Dutour 2012. Marqueurs osseux d'activités physiques: étude du squelette appendiculaire d'une population nabatéo-romaine (Syrie du Sud). Bulletin et Mémoires de la Société d'Anthropologie de Paris 24:131-151.

Benjamin, M., H. Toumi, J. Ralphs, G. Bydder, T. Best y S. Milz 2006. Where tendons and ligaments meet bone: attachment sites ('entheses') in relation to exercise and/or mechanical load. Journal of Anatomy 208:471-490.
Berato, J., O. Dutour, J. Williams, H. Zakarian y P. Acquaviva 1990. Epidémiologie des affections rhumatismales dans une population antique. Etude de la Nécropole du Haut-Empire de Saint-Lambert (Fréjus, Var). Revue du Rhumatisme 57:397-400.

Boada, A.M. 1987. Marín, un asentamiento indígena en el Valle de Samacá (Boyacá). Boletín de Arqueología-FIAN 2:27-44.

- - - 1988. La deformación craneana en Marín: un sitio del Valle de la Laguna (Samacá-Boyacá). Revista de Antropología Universidad de los Andes IV:129-141.

Bourke, J.B. 1967. A review of the paleopathology of the arthritic disease. En Diseases in Antiquity, editado por D. Brothwell y A.T. Sandison, pp. 352-370. Charles C. Thomas, Springfield, Illinois.

Bridges, P. 1991. Degenerative joint disease in hunter-gatherers and agriculturalists from the southeastern United States. American Journal of Physical Anthropology 85:379-391.

- - - 1992. Prehistoric arthritis in the Americas. Annual Review Anthropology 21:67-91.

- - - 1993. Reply to Dr Knüsel. American Journal of Physical Anthropology 91:526-527.

Brothwell, D. 1965. Digging up Bones. The Excavation, Treatment and the Study of Human Skeletal Remains. Oxford University Press, London. 
Buikstra, J. y D. Ubelaker 1994. Standars for Data Collection from Human Skeletal Remains. Arkansas Archeological Survey Research Series $N^{\circ}$ 44, Arkansas.

Campillo, D. 2001. Introducción a la Paleopatología. Bellaterra, Barcelona.

Cardoso, F.A. y C. Henderson 2010. Enthesopathy formation in the humerus: data from known age-at-death and known occupation skeletal collections. American Journal of Physical Anthropology 141:550-560.

Cohen, M. y G. Armelagos (eds.) 1984. Paleopathology at the Origins of Agriculture. Academic Press Inc, Orlando.

Cope, J., A. Berryman, D. Martin y D. Potts 2005. Robusticity and osteoarthritis at the trapeziometacarpal joint in a Bronze Age population from Tell Abraq, United Arab Emirates. American Journal of Physical Anthropology 126:391-400.

Costill, D. y J. Wilmore 2006. Physiologie du Sport et de l'Exercice: Adaptations Physiologiques à l'Exercice Physique. Traducido por A. Delamarche, P. Delamarche, C. Groussard y H. Zouhal. Cuarta edición. De Boeck Université, Bruxelles.

Crubézy, É., J. Goulet, J. Bruzek, J. Jelinek, D. Rougé y B. Ludes 2002. Epidemiology of osteoarthritis and enthesopathies in a European population dating back 7700 years. Joint Bone Spine 69:580-588.

Cunha, E. 2003. Aproximación paleopatológica a algunas enfermedades reumáticas. En Paleopatología la Enfermedad no Escrita, editado por A. Isidro y A. Malgosa, pp. 209-220. Masson, Barcelona.

Churchill, S. y A. Morris 1998. Muscle marking morphology and labour intensity in prehistoric khoisan foragers. International Journal of Osteoarchaeology 8:390-411.

Dabbs, G. 2011. Health status among prehistoric Eskimos from Point Hope, Alaska. American Journal of Physical Anthropology 146:94-103.

Dutour, O. 1986. Enthesopathies (Lesions of Muscular Insertions) as indicators of the activities of Neolithic Saharan populations. American Journal of Physical Anthropology 7:221-224.

- - - 1992. Activités physiques et squelette humain: le difficile passage de l'actuel au fossile. Bulletin et mémoires de la Société d'Anthropologie de Paris n.s.t. 4. (3-4):233-241.

Dutour, O. y Y. Ardagna 2005. La paléopathologie humaine. En Objets et Méthodes en Paléoanthropologie, editado por O. Dutour, J.-J. Hublin y B. Vandermeersch, pp. 315-341. Comité de travaux historiques et scientifiques, Paris.

Eshed, V., A. Gopher, E. Galili y I. Hershkovitz 2004. Musculoskeletal Stress Markers in Natufian hunter-gatherers and Neolithic farmers in the Levant: the upper limb. American Journal of Physical Anthropology 123:303-315.

Eshed, V., A. Gopher, R. Pinhasi e I. Hershkovitz 2010. Paleopathology and the origin of agriculture in the Levant. American Journal of Physical Anthropology 143:121-133.

Goodman, A. y D. Martin 2002. Reconstructing health profiles from skeletal remains. En The Backbone of History: Health and Nutrition in the Western Hemisphere, editado por R. Steckel y J. Rose, pp. 11-60. Cambridge University press, Cambridge.
Goodman, A., D. Martin y G. Armelagos 1984. Indicators of stress from bone and teeth. En Paleopathology at the Origins of Agriculture, editado por M. Cohen y G. Armelagos, pp. 14-38. Academic Press, Orlando.

Green, H. y J. Sutton 2001. The effects of altitude on skeletal muscle. En High Altitude: An Exploration of Human Adaptation, editado por T. Hornbein y R. Schoene, pp. 443-493. Marcel Dekker Inc, New York.

Havelková, P., S. Villotte, P. Velemínský, L. Poláček y M. Dobisíková 2011. Enthesopathies and activity patterns in the Early Medieval Great Moravian population: evidence of division of labour. International Journal of Osteoarchaeology 21:487-504.

Hawkey, D. 1998. Disability, compassion and the skeletal record: using musculoskeletal stress markers (MSM) to construct an osteobiography from Early New Mexico. International Journal of Osteoarchaeology 8:326-340.

Hawkey, D. y C. Merbs 1995. Activity-induced Musculoskeletal Stress Markers (MSM) and subsistence strategy changes among ancient Hudson Bay Eskimos. International Journal of Osteoarchaeology 5:324-338.

Henderson, C. y F. Alves-Cardoso 2013. Special issue entheseal changes and occupation: Technical and theoretical advances and their applications. International Journal of Osteoarchaeology 23:127-134.

Inoue, K., S. Hukuda, Z. Yang, M. Nakai, K. Katayama, T. Ushiyama, Y. Saruhashi, J. Huang, A. Mayeda, I. Cattedu y C. Obry 2001. Prevalence of large-joint osteoarthritis in Asian and Caucasian skeletal populations. Rheumatology 40:70-73.

Isçan, M.Y., S.R. Loth y R.K. Wright 1984. Age estimation from the rib by phase analysis: white males. Journal of Forensic Sciences 29:1094-1104.

- - - 1985. Age estimation from the rib by phase analysis: white females. Journal of Forensic Sciences 30:853-863.

Jiménez, S. y Dharmavaram 2009. Genetic aspects of familial osteoarthritis. Annals of Rheumatic Diseases 53:789-797.

Jurmain, R. 1977. Stress and the etiology of osteoarthritis. American Journal of Physical Anthropology 46:353-366.

- - - 1980. The pattern of involvement of appendicular degenerative joint disease. American Journal of Physical Anthropology 53:143-150.

- - - 1990. Paleoepidemiology of a central California prehistoric population from CA-ALA-329:II. Degenerative Disease. American Journal of Physical Anthropology 83:83-94.

Jurmain R. y L. Kilgore 1995. Skeletal evidence of osteoarthritis: a paleopathological perspective. Annals of the Rheumatic Diseases 53:443-450.

Jurmain, R. y S. Villotte 2010. Terminology. Entheses in medical literature and physical anthropology: a brief review (online). (http://www.uc.pt/en/cia/msm/MSM_terminology3 (12 julio 2012).

Kennedy, K. 1998. Markers of occupational stress: conspectus and prognosis of research. International Journal of Osteoarchaeology 8:305-310.

Kjeken, I., H. Dagfinrud, B. Slatkowsky-Christensen, P. Mowinckel, T. Uhlig, T. Kvien y A. Finset 2004. Activity 
limitations and participation restriction in women with hand osteoarthritis: patients' descriptions and associations between dimensions of functioning. Annals of the Rheumatic Diseases 64:1633-1638.

Klaus, H., C.S. Larsen y M. Tam 2009. Economic intensification and degenerative joint disease: Life and labor on the postcontact North Coast of Peru. American Journal of Physical Anthropology 139:204-221.

Knüsel, C. 1993. On the biomechanical and osteoarthritic differences between hunter-gatherers and agriculturalists. American Journal of Physical Anthropology 91:523-527.

Knüsel, C., S. Göggel y D. Lucy 1997. Comparative Degenerative Joint Disease of the vertebral column in the Medieval Monastic Cemetery of the Gilbertine Priory of St. Andrew, Fishergate, York, England. American Journal of Physical Anthropology 103:481-495.

Larsen, C.S. 1987. Bioarchaeological Interpretations of Subsistence Economy and Behavior from Human Skeletal Remains. En Advances in Archaeological Method and Theory Volume 10, editado por M. Schiffer, pp. 339-445. Academic Press, Orlando.

- - - 1999. Bioarchaeology: Interpreting Behavior from the Human Skeleton. Cambridge University Press, Cambridge.

Lieverse, A., A. Weber, V. Bazaliiskiy, O. Goriunova y N. Savel'ev 2007. Osteoarthritis in Siberia's Cis-Baikal: skeletal indicators of hunter-gatherer adaptation and cultural change. American Journal of Physical Anthropology 132:1-16.

Lovell, N. y A. Dublenko 1999. Further aspects of fur trade life depicted in the skeleton. International Journal of Osteoarchaeology 9:248-256.

Malgosa, A. 2003. Marcadores de estrés ocupacional. En Paleopatología La Enfermedad no Escrita, editado por I. Llorens y A. Malgosa, pp. 221-237. Masson, Barcelona.

Mariotti, V., F. Facchini y M. Belcastro 2004. EnthesopathiesProposal of a standardized scoring method and applications. Collegium Antropologicum 28:145-159.

- - - 2007. The study of entheses: proposal of a standardised scoring method for twenty-three entheses of the postcranial skeleton. Collegium Antropologicum 31:291-313.

Martín, J., J. Rivera-Sandoval y C. Rojas-Sepúlveda 2009. Bioarqueología. Su aporte al Proyecto Arqueológico Panamá Viejo. Canto Rodado 4:117-144.

Martínez, I. 2005. Variabilidad en el altiplano. El caso de la estatura en Soacha y Marín. Revista de Estudiantes de Arqueología:39-47.

Molnar, P. 2006. Tracing Prehistoric activities: musculoskeletal stress marker analysis of a Stone-Age population on the island of Gotland in the Baltic Sea. American Journal of Physical Anthropology 129:12-23.

- - - 2008. Patterns of physical activity and material culture on Gotland, Sweden, during the Middle Neolithic. International Journal of Osteoarchaeology 20:1-14.

Molnar, P., T. Ahlstrom y I. Leden 2011. Osteoarthritis and activity-an analysis of the relationship between eburnation, musculoskeletal stress markers (MSM) and age in two Neolithic hunter-gatherer populations from Gotland, Sweden. International Journal of Osteoarchaeology 21:283-291.
Munson Chapman, N. 1997. Evidence for Spanish influence on activity induced musculoskeletal stress markers at Pecos Pueblo. International Journal of Osteoarchaeology 7:497-506.

Nathan, H. 1962. Osteophytes of the vertebral column. The Journal of Bone and Joint Surgery 44 A(2):243-268.

Niinimäki, S. 2011. What do muscle marker ruggedness scores actually tell us? International Journal of Osteoarchaeology 21:292-299.

Norr, L. 1991. Nutritional Consequences of Prehistoric Subsistence Strategies in Lower Central America. Tesis doctoral, University of Illinois, Urbana.

Nowàcka, E. 1989. Osteología de la muestra de esqueletos de Sitio Sierra. Manuscrito en posesión del autor.

Ortner, D. 1968. Description and classification of degenerative bone changes in the distal joint surfaces of the humerus. American Journal of Physical Anthropology 28:139-156.

- - - - 2003. Identification of Pathological Conditions in Human Skeletal Remains. Academic Press, San Diego.

Oumaoui, I., S. Jiménez-Brobeil y P. Souich 2004. Markers of activity patterns in some populations of the Iberian peninsula. International Journal of Osteoarchaeology 14:343-359.

Palfi, G. y O. Dutour 1996. Activity-induced skeletal markers in historical anthropological material. International Journal of Anthropology 11:41-55.

Pechenkina, E. y M. Delgado 2006. Dimensions of health and social structure in the early intermediate period cemetery at Villa El Salvador, Peru. American Journal of Physical Anthropology 131:218-235.

Peterson, J. 1998. The Natufian hunting conundrum: spears, atlats, or bows? Musculoskeletal and armature evidence. International Journal of Osteoarchaeology 8:378-389.

Peyron, J. 1986. Osteoarthritis the epidemiologic viewpoint. Clinical Orthopaedics and Related Research 213:13-19.

Ponce, P. 2010. A Comparative Study of Activity-Related Skeletal Changes in 3rd-2nd Millenium BC Coastal Fishers and 1st Millenium AD Inland Agriculturalists in Chile, South America. Tesis doctoral. Durham University, Durham.

Resnick, D. (ed.) 2002. Diagnosis of Bone and Joint Disorders. Cuarta edición. WB Saunders Company, Philadelphia.

Rhode, M. y R. Benfer 2006. Proyecto de Investigación. Análisis de antropología física de 35 esqueletos correspondientes Ancón I (Zona Alta o Miramar). Manuscrito en posesión del autor.

Robb, J. 1994. Skeletal signs of activity in the Italian metal ages: methodological and interpretative notes. Human Evolution 9:215-229.

- - - 1998. The interpretation of skeletal muscle sites: a statistical approach. International Journal of Osteoarchaeology 8:363-377.

Robledo, B., G. Trancho y D. Campillo 1993. Factores de estrés ambiental en el hueso coxal: frecuencia de osteoartrosis en una población medieval española. Actas del II Congreso Nacional de Paleopatología, pp. 327-331, editado por J. Villalain y C. Gómez. Asociación Española de Paleopatología, Valencia.

Rodríguez, J.V. 1994. Perfil paleodemográfico muisca. El caso del cementerio de Soacha, Cundinamarca. Maguaré 10:7-36. 
- - - 1999. Los Chibchas: Pobladores Antiguos de los Andes Orientales. Aspectos Bioantropológicos. Colciencias-Universidad Nacional de Colombia, Bogotá.

- - - 2006. Las Enfermedades en las Condiciones de Vida Prehispánica de Colombia. Universidad Nacional de Colombia. Facultad de Ciencias Humanas. Departamento de Antropología, Bogotá.

Rogers, J. y P. Dieppe 1990. Skeletal palaeopathology and the rheumatic diseases: where are we now? Annals of the Rheumatic Diseases 49:885-886.

- - - 1994. Is tibiofemoral osteoarthritis in the knee joint a new disease? Annals of the Rheumatic Diseases 53:612-613.

Rogers, J., L. Shepstone y P. Dieppe 1997. Bone formers: osteophyte and enthesophyte formation are positively associated. Annals of the Rheumatic Diseases 56:85-90.

Rogers, J. y H. Waldron 1995. A Field Guide to Joint Disease in Archaeology. John Wiley and Sons Ltd, Chichester.

Rogers, J., T. Waldron y P. Dieppe 1987. Arthropaties in palaeopathology: The basis of classification according to most probable cause. Journal of Archaeological Science 14:179-193.

Rojas-Sepúlveda, C. 2005. Palepatología de Columna Vertebral con Énfasis en Estrés Ocupacional. Colección Ósea Muisca, Cementerio de Soacha (Colombia). Tesis para optar al título de Antropóloga, Universidad Nacional de Colombia, Bogotá.

- - - 2009. Relations Homme-Environnement en Amérique du Sud Précolombienne. Approche Paléoépidémiologique de la Maladie Articulaire Dégénérative et des Marqueurs Osseux d'Activités chez des Populations Anciennes des Régions Andines Septentrionnales et Panaméennes. Tesis doctoral, Université de la Méditerranée, Marseille.

Rojas-Sepúlveda, C., Y. Ardagna y O. Dutour 2008. Paleoepidemiology of vertebral degenerative disease in a PreColumbian Muisca series from Colombia. American Journal of Physical Anthropology 135:416-430.

Rojas-Sepúlveda, C. y O. Dutour 2009. Première évaluation paléoépidémiologique d'une série ostéoarchéologique de la Côte Centrale Péruvienne: Le site d'Ancón I. Bulletin et Mémoires de la Société d'Anthropologie de Paris 21:113-139.

Rosser, B. y P.W. Hochachka 1993. Metabolic capacity of muscle fibers from high-altitude natives. European Journal of applied Physiology 67:513-517.

Rothschild, B. 1997. Porosity: a curiosity without diagnostic significance. American Journal of Physical Anthropology 104:529-533.

Santos, A.L., F. Alves-Cardoso, S. Assis y S. Villotte 2010. The Coimbra workshop in Musculoskeletal Stress Markers (MSM): an annotated review (online). http://www.uc.pt/en/cia/msm/ MSM_terminology3 (12 julio 2012).

Slovak, N. 2007. Examining Imperial Influence on Perú's Central Coast: Isotopic and Cultural Analyses of Middle Horizon Burials at Ancón. Tesis doctoral, Stanford University, Stanford.

Steen, S. y R. Lane 1998. Evaluation of habitual activities among two Alaskan Eskimo populations based on musculoskeletal stress markers. International Journal of Osteoarchaeology 8:341-353.
Stefanović, S. y M. Porčić 2011. Between-group differences in the patterning of musculo-skeletal stress markers: avoiding confounding factors by focusing on qualitative aspects of physical activity. International Journal of Osteoarchaeology 23:94-105.

Stirland, A. 1998. Musculoskeletal evidence for activity: problems of evaluation. International Journal of Osteoarchaeology 8:354-362.

Suchey, J.M. y S.T. Brooks 1988. Skeletal age Determination Based on the Male os Pubis. Ponencia presentada en 12th International Conference of Anthropological and Ethnological Sciences, Zagreb.

Thillaud, P. 1992. El diagnóstico retrospectivo en paleopatología. Munibe (Antropologia-Arkeologia) Sociedad de Ciencias Arazandi, San Sebastian España Suplement 8:81-88.

- - - 1994. Lesions Osteo-Archeologiques: Recueil et Identification. Kronos B.Y. Editions, Sceaux.

Ubelaker, D.H. 1989. Human Skeletal Remains. Excavation, Analysis, Interpretation. Segunda edición, Taraxacum, Washington.

Villotte, S. 2006. Connaissances médicales actuelles, cotation des enthésopathies: nouvelle méthode. Bulletins et Mémoires de la Société d'Anthropologie de Paris 18:65-85.

- - - 2008. Enthésopathies et Activités des Hommes Préhistoriques. Recherche Méthodologique et Application aux Fossiles Européens du Paléolithique Supérieur et du Mésolithique. Tesis doctoral, Université de Bordeaux 1, Bordeaux.

Villotte, S., D. Castex, V. Couallier, O. Dutour, C. Knüsel y D. Henry-Gambier 2010. Enthesopathies as occupational stress markers: evidence from the upper limb. American Journal of Physical Anthropology 142:224-234.

Villotte, S. y C. Knüsel 2013. Understanding Entheseal Changes: Definition and life course changes. International Journal of Osteoarchaeology 23:135-146.

Waldron, H. 1991. Prevalence and distribution of osteoarthritis in a population from Georgian and early Victorian London. Annals of the Rheumatic Diseases 50:301-307.

- - - 1997. Association between osteoarhritis of the hand and knee in a population of skeletons from London. Annals of the Rheumatic Diseases 56:116-118.

Waldron T. 1992. Osteoarthritis in a Black Death Cemetery in London. International Journal of Osteoarchaeology 2:235-240.

- - - 1994. Counting the Dead: the Epidemiology of Skeletal Populations. John Wiley and Sons Ltd, Chichester.

- - - 1997. Osteoarthritis of the hip in past populations. International Journal of Osteoarchaeology 7:186-189.

- - - 2009. Paleopathology. Cambridge University press, New York.

Walker, P. y S. Hollimon 1989. Changes in osteoarthritis associated with the development of a maritime economy among Southern California Indians. International Journal of Anthropology 4:171-183.

Watkins, R. 2012. Variation in health and socioeconomic status within the W. Montague Cobb skeletal collection: Degenerative 
joint disease, trauma and cause of death. International Journal of Osteoarchaeology 22:22-44.

Weiss, E. 2003a. Effects of rowing on humeral strength. American Journal of Physical Anthropology 121:293-302.

- - - 2003b. Understanding muscle markers: aggregation and construct validity. American Journal of Physical Anthropology 121:230-240.

- - - 2004. Understanding muscle markers: lower limbs. American Journal of Physical Anthropology 125:232-238.

----2007. Muscle markers revisited: activity pattern reconstruction with controls in a Central California Amerind Population. American Journal of Physical Anthropology 133:931-940.

Weiss, E., L. Corona y B. Schultz 2012. Sex differences in musculoskeletal stress markers: problems with activity pattern reconstruction. International Journal of Osteoarchaeology 22:70-80.

Weiss, E. y R. Jurmain 2007. Osteoarthritis revisited: A contemporary review of aetiology. International Journal of Osteoarchaeology 17:437-450.

White, T. y P. Folkens 2000. Human Osteology. Segunda edición. Academic Press, San Diego.

Wilczak, C. 1998. Consideration of sexual dimorphism, age, and asymmetry in quantitative measurements of muscle insertion sites. International Journal of Osteoarchaeology 8:311-325.

Woo, E. y P. Sciulli 2011. Degenerative Joint Disease and social status in the Terminal Late Archaic Period (1000-500 B.C.) of Ohio. International Journal of Osteoarchaeology 23:529-544. 



\section{Dataciones radiocarbónicas NO deberían demorar una eternidad}

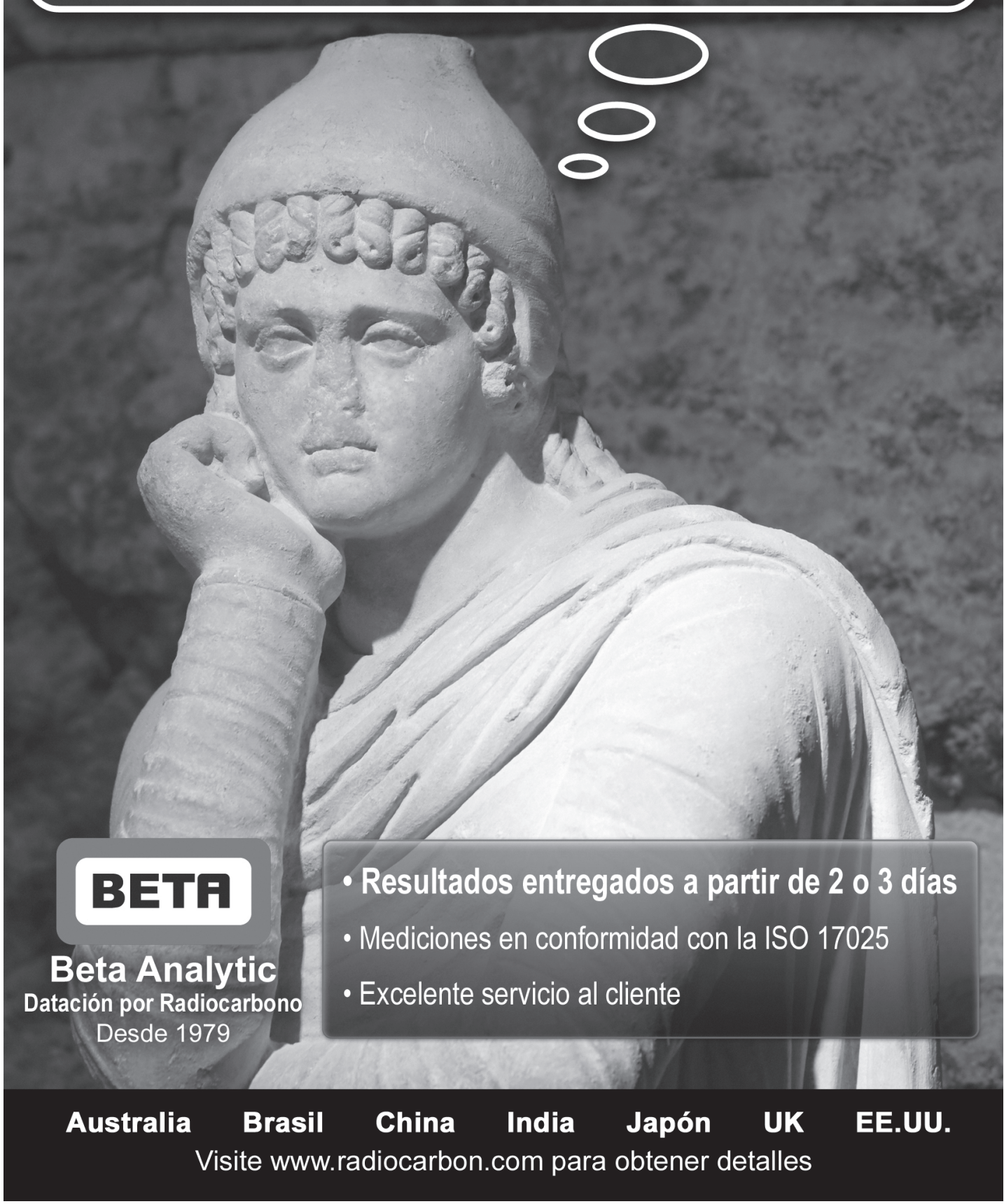


ANDROS IMPRESORES

www.androsimpresores.cl 